\title{
Self-extensions of Verma modules and differential forms on opers
}

\author{
Edward Frenkel and Constantin Teleman
}

\begin{abstract}
We compute the algebras of self-extensions of the vacuum module and the Verma modules over an affine Kac-Moody algebra $\hat{\mathfrak{g}}$ in suitable categories of Harish-Chandra modules. We show that at the critical level these algebras are isomorphic to the algebras of differential forms on various spaces of opers associated to the Langlands dual Lie algebra of $\mathfrak{g}$, whereas away from the critical level they become trivial. These results rely on and generalize the description of the corresponding algebras of endomorphisms obtained by Feigin and Frenkel and the description of the corresponding graded versions due to Fishel, Grojnowski and Teleman.
\end{abstract}

\section{Introduction}

Let $\mathfrak{g}$ be a simple finite-dimensional Lie algebra and $\hat{\mathfrak{g}}_{\kappa}$, where $\kappa$ is an invariant inner product on $\mathfrak{g}$, the corresponding affine Kac-Moody algebra. Consider the vacuum module $\mathbb{V}_{\kappa}$ over $\hat{\mathfrak{g}}_{\kappa}$ (see $\S 2$ for the precise definitions). According to the results of [FF92, Fre04], the algebra of endomorphisms of $\mathbb{V}_{\kappa}$ is trivial, i.e. isomorphic to $\mathbb{C}$, unless $\kappa=\kappa_{\mathrm{c}}$, the critical value. In contrast, the algebra $\operatorname{End}_{\hat{\mathfrak{g}}_{\kappa_{\mathrm{c}}}} \mathbb{V}_{\kappa_{\mathrm{C}}}$ is large and is in fact canonically isomorphic to the algebra of functions on the space $\mathrm{Op}_{L} \mathfrak{g}(D)$ of ${ }^{L} \mathfrak{g}$-opers on the disc, where ${ }^{L} \mathfrak{g}$ is the Lie algebra that is Langlands dual to $\mathfrak{g}$ (its Cartan matrix is the transpose of that of $\mathfrak{g}$ ).

In this paper we consider the algebra of endomorphisms of $\mathbb{V}_{\kappa}$ in the derived category of $\left(\hat{\mathfrak{g}}_{\kappa}, G[[t]]\right)$-modules, or in other words the algebra of self-extensions of $\mathbb{V}_{\kappa}$ in the abelian category of $\left(\hat{\mathfrak{g}}_{\kappa}, G[[t]]\right)$-modules (here $G$ is the connected simply connected algebraic group corresponding to $\mathfrak{g})$. As we show in $\S 2$, this algebra may be realized as the relative cohomology

$$
H^{\bullet}\left(\mathfrak{g}((t)), \mathfrak{g}, \operatorname{End}_{\mathbb{C}} \mathbb{V}_{\kappa}\right) \simeq H^{\bullet}\left(\mathfrak{g}[[t]], \mathfrak{g}, \mathbb{V}_{\kappa}\right)
$$

We show that for $\kappa \neq \kappa_{\mathrm{c}}$ we have $H^{i}\left(\mathfrak{g}((t)), \mathfrak{g}\right.$, End $\left._{\mathbb{C}} \mathbb{V}_{\kappa}\right)=0$ if $i>0$, so the corresponding algebra of self-extensions of $\mathbb{V}_{\kappa}$ is isomorphic to $\mathbb{C}$. But if $\kappa=\kappa_{\mathrm{c}}$, then this algebra is isomorphic to the algebra of differential forms on $\mathrm{Op}_{L_{\mathfrak{g}}}(D)$ (more precisely, this isomorphism is defined up to a scalar which is fixed once we choose an invariant bilinear form $\kappa_{0}$ on $\mathfrak{g}$ ). Moreover, deforming $\mathbb{V}_{\kappa_{\mathrm{c}}}$ to $\mathbb{V}_{\kappa}$, we obtain a differential on the cohomology $H^{\bullet}\left(\mathfrak{g}((t)), \mathfrak{g}, \operatorname{End}_{\mathbb{C}} \mathbb{V}_{\kappa_{\mathrm{c}}}\right)$ which coincides with the de Rham differential.

In order to prove this result, we use the quasi-classical statement about the cohomology $H^{\bullet}\left(\mathfrak{g}[[t]], \mathfrak{g}, \mathbb{V}^{\mathrm{cl}}\right)$, where $\mathbb{V}^{\mathrm{cl}}$ is the associate graded of $\mathbb{V}_{\kappa_{\mathrm{c}}}$ with respect to the PBW filtration. The space $\mathrm{Op}_{L_{\mathfrak{g}}}(D)$ is an affine space modeled on the vector space $C_{\mathfrak{g}^{*}, \omega}$ defined in $\S 1$. According to $\left[\right.$ FGT04], $H^{\bullet}\left(\mathfrak{g}[[t]], \mathfrak{g}, \mathbb{V}^{\mathrm{cl}}\right)$ is isomorphic to the algebra of differential forms on $C_{\mathfrak{g}^{*}, \omega}$. Using this result and the description of $H^{0}\left(\mathfrak{g}[[t]], \mathfrak{g}, \mathbb{V}_{\kappa_{\mathrm{c}}}\right)$ from [FF92] and [Fre04] mentioned above, we

Received 7 May 2004, accepted in final form 16 September 2005.

2000 Mathematics Subject Classification 17B67 (primary), 17B56 (secondary).

Keywords: affine Kac-Moody algebra, critical level, Verma module, oper.

This journal is (C) Foundation Compositio Mathematica 2006. 


\section{E. Frenkel and C. Teleman}

obtain our result by employing the spectral sequence on the cohomology corresponding to the PBW filtration.

We also prove an analogue of this result for the Verma modules $\mathbb{M}_{\lambda, \kappa}$. In this case we consider the algebra of endomorphisms of $\mathbb{M}_{\lambda, \kappa}$ in the derived category of $\left(\hat{\mathfrak{g}}_{\kappa}, \widetilde{B}\right)$ Harish-Chandra modules, where $\widetilde{B}$ is the Iwahori subgroup of $G[[t]]$, the preimage of a Borel subgroup $B \subset G$ under the evaluation homomorphism $G[[t]] \rightarrow G$. This algebra is realized as the cohomology

$$
H^{\bullet}\left(\mathfrak{g}((t)), \mathfrak{h}, \operatorname{End}_{\mathbb{C}} \mathbb{M}_{\lambda, \kappa}\right) \simeq H^{\bullet}\left(\widetilde{\mathfrak{b}}, \mathfrak{h}, \mathbb{M}_{\lambda, \kappa} \otimes \mathbb{C}_{-\lambda}\right),
$$

where $\widetilde{\mathfrak{b}}$ is the Lie algebra of $\widetilde{B}$ and $\mathfrak{h}$ is the Cartan subalgebra of the constant subalgebra $\mathfrak{g}$. It follows from the results of [FF92] and [Fre04] that $\operatorname{End}_{\hat{\mathfrak{g}}_{\kappa_{\mathrm{c}}}} \mathbb{M}_{\lambda, \kappa_{\mathrm{c}}}$ is the algebra of functions on the space $\mathrm{Op}_{L_{\mathfrak{g}}}(D)_{\lambda}$ of ${ }^{L} \mathfrak{g}$-opers on $D$ with regular singularities and residue $-\lambda-\rho$. We show, in the same way as in the case of the vacuum modules, that the full algebra of self-extensions of $\mathbb{M}_{\lambda, \kappa_{\mathrm{c}}}$ is isomorphic to the algebra of differential forms on $\mathrm{Op}_{L_{\mathfrak{g}}}(D)_{\lambda}$. We also show that for $\kappa \neq \kappa_{\mathrm{c}}$ this algebra is just isomorphic to $\mathbb{C}$.

The above statements are closely related to and were motivated by the result of $\mathrm{B}$. Feigin announced in [Fei91] that the cohomology of $\hat{\mathfrak{g}}_{\kappa_{\mathrm{c}}}$ with coefficients in the completion of the enveloping algebra of $\hat{\mathfrak{g}}_{\kappa_{\mathrm{c}}}$ (with respect to the adjoint action) is isomorphic to the algebra of differential forms on the space of ${ }^{L} \mathfrak{g}$-opers on the punctured disc.

The paper is organized as follows. In $\S 1$ we consider the graded version of the vacuum module (we call it 'classical') and recall the result of [FGT04]. In $\S 2$ we define the vacuum module $\mathbb{V}_{\kappa}$ over the affine Kac-Moody algebra $\hat{\mathfrak{g}}_{\kappa}$ and describe the algebra of its self-extensions in a suitable category of Harish-Chandra modules as the relative Lie algebra cohomology $H^{\bullet}\left(\mathfrak{g}((t)), \mathfrak{g}\right.$, End $\left._{\mathbb{C}} \mathbb{V}_{\kappa}\right)$. In $\S 3$ we realize it as the cohomology of the Chevalley complex of $\mathfrak{g}[[t]]$ relative to $\mathfrak{g}$ with coefficients in $\mathbb{V}_{\kappa}$. We show that the algebra structure on this cohomology is induced by a DG vertex superalgebra structure on the complex. Next, we show that the latter is actually skew-commutative, and hence gives rise to the structure of skew-commutative associative algebra on $H^{\bullet}\left(\mathfrak{g}[[t]], \mathbb{V}_{\kappa_{\mathrm{c}}}\right)$. Our first proof of this fact was based on an explicit computation of this cohomology. But, as was subsequently pointed out to us by D. Gaitsgory, this is in fact a corollary of a general property of the Chevalley complex of an arbitrary vertex Lie superalgebra (see Proposition 3.3 and Remark 3.5).

In $\S 4$ we compute the cohomology of the vacuum module $\mathbb{V}_{\kappa_{\mathrm{c}}}$ and show that it is isomorphic to the algebra of differential forms on the space $\mathrm{Op}_{L_{\mathfrak{g}}}(D)$. We then show that the cohomology of $\mathbb{V}_{\kappa}$ where $\kappa \neq \kappa_{\mathrm{c}}$ is isomorphic to $\mathbb{C}$. In $\S 5$ we consider the graded version $\mathbb{M}^{\text {cl }}$ of the Verma module and compute the cohomology $H^{\bullet}\left(\widetilde{\mathfrak{b}}, \mathfrak{h}, \mathbb{M}^{\mathrm{cl}}\right)$ using the results of [FGT04]. Finally, in $\S 6$ we use the computation in the graded case and a description of the algebra of endomorphisms of the Verma modules $\mathbb{M}_{\lambda, \kappa}$ to find the full algebra of self-extensions of $\mathbb{M}_{\lambda, \kappa}$ in the appropriate category of Harish-Chandra modules.

\section{Cohomology of the classical vacuum module}

Let $\mathfrak{g}$ be a simple finite-dimensional Lie algebra and $G$ the connected simply connected algebraic group corresponding to $\mathfrak{g}$. Introduce the 'classical' vacuum module over the Lie algebra $\mathfrak{g}[[t]]$,

$$
\mathbb{V}^{\mathrm{cl}}=\operatorname{Sym}(\mathfrak{g}((t)) / \mathfrak{g}[[t]]) \simeq \operatorname{Fun}\left(\mathfrak{g}^{*}[[t]] d t\right),
$$

where we use the residue pairing. We consider the relative cohomology $H^{\bullet}\left(\mathfrak{g}[[t]], \mathfrak{g}, \mathbb{V}^{\text {cl }}\right)$ of $\mathfrak{g}[[t]]$ modulo the constant Lie subalgebra $\mathfrak{g}$ with coefficients in $\mathbb{V}^{\text {cl }}$. This cohomology may be computed by the standard Chevalley complex of Lie algebra cohomology

$$
C^{\bullet}\left(\mathfrak{g}[[t]], \mathfrak{g}, \mathbb{V}^{\mathrm{cl}}\right)=\left(\mathbb{V}^{\mathrm{cl}} \otimes \bigwedge^{\bullet}(\mathfrak{g}[[t]] / \mathfrak{g})^{*}\right)^{\mathfrak{g}}=\left(\operatorname{Fun}\left(\mathfrak{g}^{*}[[t]] d t\right) \otimes \bigwedge^{\bullet}(\mathfrak{g}[[t]] / \mathfrak{g})^{*}\right)^{\mathfrak{g}} .
$$




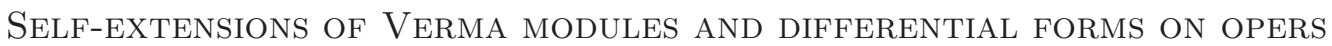

Remark 1.1. Here and below we view $\mathfrak{g}[[t]]$ and similar vector spaces as complete topological spaces, and by the dual of such a vector space we mean the vector space of continuous linear functionals on it (the topological dual). As the result, $C^{\bullet}\left(\mathfrak{g}[[t]], \mathfrak{g}, \mathbb{V}^{\mathrm{cl}}\right)$ is a vector space topologized as the direct limit of its finite-dimensional subspaces.

The commutative algebra structure on $\mathbb{V}^{\text {cl }}$ gives rise to a skew-commutative algebra structure on the complex $C^{\bullet}\left(\mathfrak{g}[[t]], \mathfrak{g}, \mathbb{V}^{\mathrm{cl}}\right)$. The Chevalley differential is a derivation of this algebra structure. Hence the cohomology $H^{\bullet}\left(\mathfrak{g}[[t]], \mathfrak{g}, \mathbb{V}^{\text {cl }}\right)$ also acquires the structure of a skew-commutative algebra. We recall the description of this algebra obtained in [FGT04].

Set

$$
C_{\mathfrak{g}^{*}}=\mathfrak{g}^{*} / G:=\operatorname{Spec}\left(\text { Fun } \mathfrak{g}^{*}\right)^{G}
$$

and define the local Hitchin space $C_{\mathfrak{g}^{*}, \omega}$ as

$$
C_{\mathfrak{g}^{*}, \omega}=\Gamma\left(D, \omega \underset{\mathbb{C}^{\times}}{\times} C_{\mathfrak{g}^{*}}\right)
$$

where $\omega$ is the canonical line bundle on the disc $D=\operatorname{Spec} \mathbb{C}[[t]]$. Let us choose generators $P_{i}, i=$ $1, \ldots, \ell$, of $\left(\text { Fun } \mathfrak{g}^{*}\right)^{G}$ of degrees $d_{i}+1$, where the $d_{i}$ are the exponents of $\mathfrak{g}$, so that $\left(\text { Fun } \mathfrak{g}^{*}\right)^{G}=$ $\mathbb{C}\left[P_{i}\right]_{i=1, \ldots, \ell}$. Then we obtain an identification

$$
\operatorname{Fun}\left(C_{\mathfrak{g}^{*}, \omega}\right)=\mathbb{C}\left[P_{i, n}\right]_{i=1, \ldots, \ell ; n \geqslant 0},
$$

where the $P_{i, n}$ are the functions on $\mathfrak{g}^{*}[[t]] d t$ defined by the formula

$$
P_{i, n}(f(t) d t)=\text { the } t^{n} \text {-coefficient of } P_{i}(f(t)) \text {. }
$$

Note that this definition depends on the choice of $t$, but we obtain a coordinate-independent isomorphism

$$
C_{\mathfrak{g}^{*}, \omega} \simeq \bigoplus_{i=1}^{\ell} \Gamma\left(D, \omega^{\otimes\left(d_{i}+1\right)}\right)=\mathbb{C}[[t]](d t)^{\otimes\left(d_{i}+1\right)}
$$

(it depends only on the choice of the generators $P_{i}$ in $\left(\text { Fun } \mathfrak{g}^{*}\right)^{G}$ ).

It is clear that the functions $P_{i, n}$ on $\mathfrak{g}^{*}[[t]] d t$ are $\mathfrak{g}[[t]]$-invariant. Hence we obtain a homomorphism

$$
\operatorname{Fun}\left(C_{\mathfrak{g}^{*}, \omega}\right) \rightarrow H^{0}\left(\mathfrak{g}[[t]], \mathfrak{g}, \mathbb{V}^{\mathrm{cl}}\right) .
$$

Next, following [FGT04], we construct a map

$$
\varphi_{\kappa_{0}}^{\mathrm{cl}}: \operatorname{Fun}\left(C_{\mathfrak{g}^{*}, \omega}\right) \rightarrow H^{1}\left(\mathfrak{g}[[t]], \mathfrak{g}, \mathbb{V}^{\mathrm{cl}}\right),
$$

for any non-zero invariant inner product $\kappa_{0}$ on $\mathfrak{g}$ (recall that such $\kappa_{0}$ is unique up to a scalar). By abusing notation, we will also denote by $\kappa_{0}$ the corresponding map $\mathfrak{g}^{*} \rightarrow \mathfrak{g}$. We need to associate to each $P \in \operatorname{Fun}\left(C_{\mathfrak{g}^{*}, \omega}\right)$ a cocycle $\varphi_{\kappa_{0}}^{\text {cl }}(P)$ in

$$
C^{1}\left(\mathfrak{g}[[t]], \mathfrak{g}, \mathbb{V}^{\mathrm{cl}}\right)=\operatorname{Hom}\left(\mathfrak{g}[[t]] / \mathfrak{g}, \operatorname{Fun}\left(\mathfrak{g}^{*}[[t]] d t\right)\right)^{\mathfrak{g}} .
$$

We set

$$
\left(\varphi_{\kappa_{0}}^{\mathrm{cl}}(P)\right)(x(t))=\partial_{\kappa_{0}(d x(t))} \cdot P=\left\langle\kappa_{0}(d x(t)), d P\right\rangle
$$

where $\kappa_{0}(d x(t)) \in \mathfrak{g}^{*}[[t]] d t$ is considered as the constant tangent vector field to $\mathfrak{g}^{*}[[t]] d t$, and $\partial_{\kappa_{0}(d x(t))}$ is the corresponding directional derivative. It is clear from this formula that $\varphi_{\kappa_{0}}^{\mathrm{cl}}$ factors through the de Rham differential $d: \operatorname{Fun}\left(C_{\mathfrak{g}^{*}, \omega}\right) \rightarrow \Omega^{1}\left(C_{\mathfrak{g}^{*}, \omega}\right)$.

In particular, applying the map $\varphi_{\kappa_{0}}^{\mathrm{cl}}$ to the space $C_{\mathfrak{g}^{*}, \omega}^{*}$ of generators of Fun $\left(C_{\mathfrak{g}^{*}, \omega}\right)$, which is the dual space to $C_{\mathfrak{g}^{*}, \omega}$, we obtain a map

$$
C_{\mathfrak{g}^{*}, \omega}^{*} \rightarrow H^{1}\left(\mathfrak{g}[[t]], \mathfrak{g}, \mathbb{V}^{\mathrm{cl}}\right) .
$$




\section{E. Frenkel and C. Teleman}

If we multiply $\kappa_{0}$ by $\lambda$, then this map will get divided by $\lambda$.

The following theorem is a version of Theorem B of [FGT04].

ThEOREM 1.2. There is an isomorphism of graded algebras

$$
H^{\bullet}\left(\mathfrak{g}[[t]], \mathfrak{g}, \mathbb{V}^{\mathrm{cl}}\right) \simeq \Omega^{\bullet}\left(C_{\mathfrak{g}^{*}, \omega}\right)=\operatorname{Fun}\left(C_{\mathfrak{g}^{*}, \omega}\right) \otimes \bigwedge^{\bullet}\left(C_{\mathfrak{g}^{*}, \omega}^{*}\right) .
$$

The right-hand side is a free skew-commutative algebra with even generators $P_{i, n} \in H^{0}\left(\mathfrak{g}[[t]], \mathfrak{g}, \mathbb{V}^{\mathrm{cl}}\right)$ given by formula (1.1) and odd generators $\varphi_{\kappa_{0}}^{\mathrm{cl}}\left(P_{i, n}\right) \in H^{1}\left(\mathfrak{g}[[t]], \mathfrak{g}, \mathbb{V}^{\mathrm{cl}}\right)$ given by formula (1.2).

We will now explain how to obtain the odd generators from the even ones by using a deformation of the $\mathfrak{g}[[t]]$-module $\mathbb{V}^{\mathrm{cl}}$.

For $h \in \mathbb{C}$, let $\mathrm{Conn}_{h}$ be the space of $h$-connections on the trivial $G$-bundle on the disc $D$. These are operators of the form $h \partial_{t}+A(t)$, where $A(t) d t$ is a section of $\mathfrak{g} \otimes \omega$ on $D$. The Lie algebra $\mathfrak{g}[[t]]$ acts on this space by infinitesimal gauge transformations:

$$
x(t) \cdot A(t)=[x(t), A(t)]-h \partial_{t} x(t) .
$$

In particular, Conn ${ }_{h}$ becomes the space $\mathfrak{g}[[t]] d t$ when $h=0$. We set

$$
\mathbb{V}_{h}^{\mathrm{cl}}=\operatorname{Fun}\left(\operatorname{Conn}_{h}\right) \text {. }
$$

Recall that $\mathbb{V}^{\mathrm{cl}}=\operatorname{Fun}\left(\mathfrak{g}^{*}[[t]] d t\right)$. Using the invariant inner product $\kappa_{0}$ on $\mathfrak{g}$, we identify $\mathfrak{g}^{*}$ with $\mathfrak{g}$ and hence $\mathbb{V}^{\mathrm{cl}}$ with Fun $(\mathfrak{g}[[t]] d t)$. Then $\mathbb{V}_{h}^{\text {cl }}$ becomes a one-parameter deformation of $\mathbb{V}^{\mathrm{cl}}$. Note that the spaces $\mathrm{Conn}_{h}$ are isomorphic to each other for all non-zero values of $h$, and so are the modules $\mathbb{V}_{h}^{c l}$.

Explicitly, the action (1.3) translates into the following action of $\mathfrak{g}[[t]]$ on $\mathbb{V}_{h}^{\text {cl }}$. For $A \in \mathfrak{g}, n \in \mathbb{Z}$, denote $A \otimes t^{n}$ by $A_{n}$. Then the difference between the actions of $A_{n} \in \mathfrak{g}[[t]]$ on monomial elements $B_{1, m_{1}} \cdots B_{k, m_{k}}, m_{i}<0$, in $\mathbb{V}_{h}^{c l}$ and $\mathbb{V}^{\text {cl }}$ is equal to

$$
h \sum_{i=1}^{k} n \delta_{n,-m_{i}} \kappa_{0}\left(A, B_{i}\right) B_{1, m_{1}} \cdots \widehat{B}_{i, m_{i}} \cdots B_{k, m_{k}} .
$$

When $h \neq 0$, any $h$-connection can be brought to the form $h \partial_{t}$ by using gauge transformations from the first congruence subgroup

$$
G^{(1)}=\{g \in G[[t]] \mid g(0)=1\} .
$$

Therefore the Lie algebra $\mathfrak{g} \otimes t \mathbb{C}[[t]]$ acts co-freely on $\mathbb{V}_{h}^{\text {cl }}$ and we obtain the following result.

Lemma 1.3. For $h \neq 0$ the cohomology $H^{i}\left(\mathfrak{g}[[t]], \mathfrak{g}, \mathbb{V}_{h}^{\mathrm{cl}}\right)$ vanishes for $i>0$, and $H^{0}\left(\mathfrak{g}[[t]], \mathfrak{g}, \mathbb{V}_{h}^{\mathrm{cl}}\right)=\mathbb{C}$.

The one-parameter family of $\mathfrak{g}[[t]]$-modules $\mathbb{V}_{h}^{\text {cl }}$ gives rise to a $\mathfrak{g}[[t]] \otimes \mathbb{C}[h]$-module, free over $\mathbb{C}[h]$, which by abuse of notation we also denote by $\mathbb{V}_{h}^{c l}$. Consider the Chevalley complex $C^{\bullet}\left(\mathfrak{g}[[t]], \mathfrak{g}, \mathbb{V}_{h}^{\mathrm{cl}}\right)$ of relative cohomology of $\mathfrak{g}[[t]]$ modulo $\mathfrak{g}$ with coefficients in this module. Given a class $\omega$ in $H^{i}\left(\mathfrak{g}[[t]], \mathfrak{g}, \mathbb{V}^{\mathrm{cl}}\right)$, we choose a cocycle $\widetilde{\omega}$ representing it and extend it in an arbitrary way to an element $\widetilde{\omega}(h)$ of $C^{\bullet}\left(\mathfrak{g}[[t]], \mathfrak{g}, \mathbb{V}_{h}^{\text {cl }}\right)$. Applying the differential of this complex to $\widetilde{\omega}(h)$, dividing by $h$ and considering the result modulo $h$, we obtain an element of $C^{i+1}\left(\mathfrak{g}[[t]], \mathfrak{g}, \mathbb{V}^{\mathrm{cl}}\right)$. It is clear that this element is a cocycle and that the corresponding cohomology class in $H^{i+1}\left(\mathfrak{g}[[t]], \mathfrak{g}, \mathbb{V}^{\mathrm{cl}}\right)$ is independent of all choices. Thus, we obtain well-defined linear maps

$$
\varphi_{\kappa 0}^{\mathrm{cl}, i}: H^{i}\left(\mathfrak{g}[[t]], \mathfrak{g}, \mathbb{V}^{\mathrm{cl}}\right) \rightarrow H^{i+1}\left(\mathfrak{g}[[t]], \mathfrak{g}, \mathbb{V}^{\mathrm{cl}}\right)
$$

for all $i \geqslant 0$. Writing down the definition of $\varphi_{\kappa_{0}}^{\mathrm{cl}, 0}$ explicitly, using formula (1.4), we obtain that $\varphi_{\kappa_{0}}^{\mathrm{cl}, 0}$ acts on $H^{0}\left(\mathfrak{g}[[t]], \mathfrak{g}, \mathbb{V}^{\mathrm{cl}}\right)$ precisely as the map $\varphi_{\kappa_{0}}^{\mathrm{cl}}$ given by formula $(1.2)$. 


\section{SELF-EXTENSIONS OF VERMa MOdUles AND DifFERENTIAL FORMS ON OPERS}

The differential of the Chevalley complex $C^{\bullet}\left(\mathfrak{g}[[t]], \mathfrak{g}, \mathbb{V}_{h}^{\text {cl }}\right)$ is an odd derivation of its algebra structure. Therefore we obtain that the operators $\varphi_{\kappa 0}^{\text {cl, } i}$ combine into an odd derivation of the algebra $H^{\bullet}\left(\mathfrak{g}[[t]], \mathfrak{g}, \mathbb{V}^{\mathrm{cl}}\right)$, which we will also denote by $\varphi_{\kappa_{0}}^{\mathrm{cl}}$. We have $\varphi_{\kappa_{0}}^{\mathrm{cl}, i+1} \circ \varphi_{\kappa_{0}}^{\mathrm{cl}, i}=0$ for all $i \geqslant 0$.

Recall from Theorem 1.2 that we have an isomorphism (dependent on $\kappa_{0}$ )

$$
H^{\bullet}\left(\mathfrak{g}[[t]], \mathfrak{g}, \mathbb{V}^{\mathrm{cl}}\right) \simeq \Omega^{\bullet}\left(C_{\mathfrak{g}^{*}, \omega}\right),
$$

where $\Omega^{\bullet}\left(C_{\mathfrak{g}^{*}, \omega}\right)$ is the algebra of differential forms on $C_{\mathfrak{g}^{*}, \omega}$. Under this identification the derivation $\varphi_{\kappa_{0}}^{\mathrm{cl}}$ becomes nothing but the de Rham differential.

\section{Generalities on extensions}

Fix an invariant inner product $\kappa$ on $\mathfrak{g}$ (recall that it is unique up to a scalar), and let $\hat{\mathfrak{g}}_{\kappa}$ denote the one-dimensional central extension of $\mathfrak{g} \otimes \mathbb{C}((t))$,

$$
0 \rightarrow \mathbb{C} K \rightarrow \hat{\mathfrak{g}}_{\kappa} \rightarrow \mathfrak{g} \otimes \mathbb{C}((t)) \rightarrow 0
$$

with the commutation relations

$$
[A \otimes f(t), B \otimes g(t)]=[A, B] \otimes f(t) g(t)-(\kappa(A, B) \operatorname{Res} f d g) K,
$$

where $K$ is a central element. The Lie algebra $\hat{\mathfrak{g}}_{\kappa}$ is the affine Kac-Moody algebra associated to $\kappa$.

Introduce the vacuum module of level $\kappa$ :

$$
\mathbb{V}_{\kappa}=\operatorname{Ind}_{\mathfrak{g}[[t]] \oplus \mathbb{C} K}^{\hat{\mathfrak{g}}_{\kappa}} \mathbb{C}
$$

where $\mathfrak{g}[[t]]$ acts on the one-dimensional space $\mathbb{C}$ by 0 , and $K$ as the identity. We denote by $v$ the generating vector of this module.

We wish to compute the algebra $\operatorname{Ext}^{\bullet}\left(\mathbb{V}_{\kappa}, \mathbb{V}_{\kappa}\right)$ of self-extensions of $\mathbb{V}_{\kappa}$ in suitable categories of Harish-Chandra modules (see Propositions 2.1 and 2.2 below), where the multiplication is the Yoneda product. In this section we will show that this algebra is given by the cohomologies of a suitable relative Chevalley complex. In $\S 3$ we will show that this algebra is skew-commutative. Then we will compute this algebra in $\S 4$.

In what follows by a representation of the group $G[[t]]$ we will understand a direct limit of its finite-dimensional algebraic representations. In particular, any vector $v$ in such a representation is invariant under the $N$ th congruence subgroup of $G[[t]]$ for sufficiently large $N$ and hence satisfies $t^{N} \mathfrak{g}[[t]] v=0$. Let $\operatorname{Rep} G[[t]]$ be the category of all such representations.

Consider the category $H C\left(\hat{\mathfrak{g}}_{\kappa}, G[[t]]\right)$ of Harish-Chandra modules for the pair $\left(\hat{\mathfrak{g}}_{\kappa}, G[[t]]\right)$, i.e. $\hat{\mathfrak{g}}_{\kappa^{-}}$ modules on which the action of $\mathfrak{g}[[t]]$ exponentiates to an action of $G[[t]]$, and such that $K$ acts as the identity. This is a full additive subcategory within all $\hat{\mathfrak{g}}_{\kappa}$-modules, closed under forming kernels and cokernels, and is therefore abelian.

Next, we consider the relative Chevalley complex of continuous, $\mathfrak{g}$-invariant linear maps from $\bigwedge \mathfrak{g}((t)) / \mathfrak{g}$ to End $\mathbb{V}_{\kappa}$. Here End $\mathbb{V}_{\kappa}$ is topologized as

$$
\stackrel{\lim }{\longleftarrow} \operatorname{Hom}\left(V_{\alpha}, \mathbb{V}_{\kappa}\right), \quad \text { where } \quad \mathbb{V}_{\kappa}=\underset{\lim }{\longrightarrow} V_{\alpha}, \quad \operatorname{dim} V_{\alpha}<\infty
$$

This complex is naturally a DG algebra, so its cohomology $H^{\bullet}\left(\mathfrak{g}((t)), \mathfrak{g}\right.$, End $\left.\mathbb{V}_{\kappa}\right)$ acquires the structure of an associative (super)algebra.

Proposition 2.1. There is a natural isomorphism

$$
\operatorname{Ext}_{H C\left(\hat{\mathfrak{g}}_{\kappa}, G[[t]]\right)}^{\bullet}\left(\mathbb{V}_{\kappa}, \mathbb{V}_{\kappa}\right) \simeq H^{\bullet}\left(\mathfrak{g}((t)), \mathfrak{g}, \text { End } \mathbb{V}_{\kappa}\right) .
$$

Moreover, the Yoneda product corresponds to the cup-product on the Chevalley complex. 


\section{E. Frenkel and C. Teleman}

As $H C\left(\hat{\mathfrak{g}}_{\kappa}, G[[t]]\right)$ does not have enough projectives or injectives, this requires an argument.

Proof. The cohomologies of the Chevalley complex of continuous, $G$-invariant linear maps $M \otimes$ $\wedge \mathfrak{g}((t)) / \mathfrak{g} \rightarrow N$ define the bi-additive functors

$$
(M, N) \mapsto \mathrm{E}^{k}(M, N) .
$$

The $\mathrm{E}^{\bullet}$ are equipped with functorial 'connecting homomorphisms', converting short exact sequences, in either variable, into long exact sequences. This situation is summarized by saying that the $\mathrm{E}^{\bullet}$ form an exact connected right sequence of functors, in each variable separately [Str78, III, §2], [Lan84, $\mathrm{IV}, \S 7]$. Note that $\mathrm{E}^{0}=\mathrm{Hom}$, which is left exact.

The Yoneda Ext, on the other hand, are the universal exact connected right sequences starting with Hom [Str78, III, $\S 1]$; they therefore map functorially to the $\mathrm{E}^{\bullet}$. To show equality of the two functors, it suffices to check universality of the $\mathrm{E}^{\bullet}$. This will follow from Grothendieck's erasability criterion [Str78, Theorem 3.4.3], but first we wish to spell out our map from Ext to E.

Consider first $k=1$. A representative $N \rightarrow X(\eta) \rightarrow M$ of a class $\eta \in$ Ext $^{1}$ gives, after a choice of $G$-invariant linear splitting, a crossed homomorphism $\mathfrak{g}((t)) \times M \rightarrow N$. The latter is the same as a one-cocycle in the relative Chevalley complex. Changing the splitting modifies the cocycle by a coboundary, so we get a well-defined Chevalley class. Note that our cocycle will be continuous, because any linear splitting of $X(\eta)$ is so. ${ }^{1}$ It is a standard exercise to check additivity and functoriality of the resulting map $\mathrm{Ext}^{1} \rightarrow \mathrm{E}^{1}$, along with the match of the first connecting maps in the long exact sequence.

A Yoneda $k$-extension $N \rightarrow X^{1} \rightarrow X^{2} \rightarrow \cdots \rightarrow X^{k} \rightarrow M$ factors into a product of 1-extensions, by an epic-monic factorization (i.e. representing each morphism $X^{p} \rightarrow X^{p+1}$ as the composition $\left.X^{p} \rightarrow \operatorname{Im} X^{p} \rightarrow X^{p+1}\right)$. We chose $G$-linear splittings of the short exact sequences

$$
0 \rightarrow \operatorname{Im} X^{p-1} \rightarrow X^{p} \rightarrow \operatorname{Im} X^{p} \rightarrow 0
$$

to obtain Chevalley one-cocycles, and assign to the extension $\left(X^{\bullet}\right)$ the cup-product of the corresponding classes, obtained from the previous construction. Clearly, that is independent of the splittings, but we must check that this assignment descends to equivalence classes. That is, for a morphism (commutative diagram) of $k$-extensions

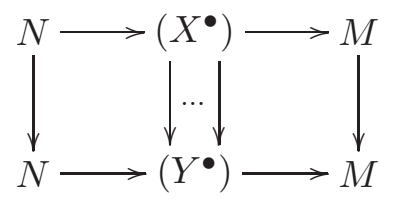

the associated Chevalley classes agree. To see that, note that we can construct commuting splittings of the two extensions (by compatible splitting of each step in their epic-monic factorizations). The associated Chevalley $(M, N)$-cocycles then agree, by commutativity of the diagram; so our assignment of Chevalley classes descends to Yoneda equivalence classes.

We now have a transformation of functors which preserves the products, and must check it is an isomorphism. We say that the functors $\mathrm{E}^{\bullet}(M, N)$ are erasable in $M$ if, for any $k>0$ and $\eta \in \mathrm{E}^{k}(M, N)$, there exists an epimorphism $f: M^{\prime} \rightarrow M$ so that $f^{*} \eta=0$ in $\mathrm{E}^{k}\left(M^{\prime}, N\right)$. The Ext ${ }^{\bullet}$ always satisfy this condition, essentially by construction (take $M^{\prime}=X^{k}$ above). Assuming erasability of $\mathrm{E}^{\bullet}$, let us check that our transformation $\Phi: \mathrm{Ext}^{\bullet} \rightarrow \mathrm{E}^{\bullet}$ is an isomorphism, by induction on $k$; we know it for $k=0$. Let then $\eta \in \operatorname{ker} \Phi: \operatorname{Ext}^{k}(M, N) \rightarrow \mathrm{E}^{k}(M, N)$ and assume that $f^{*} \eta=0$ as above. With $K=\operatorname{ker} f$, it follows that $\eta=\delta \zeta$ for some $\zeta \in \operatorname{Ext}^{k-1}(K, N)$.

\footnotetext{
${ }^{1}$ It is not difficult to show that our Harish-Chandra category is closed under forming extensions, whence it follows that all one-cocycles are in fact continuous.
} 


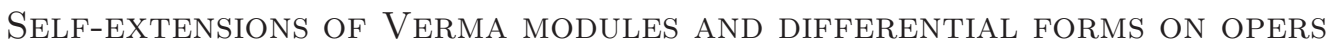

Then, $\delta \Phi(\zeta)=\Phi(\eta)=0$, so $\Phi(\zeta)$ comes from $\mathrm{E}^{k-1}\left(M^{\prime}, N\right)$. By inductive assumption, the latter is $\operatorname{Ext}^{k-1}\left(M^{\prime}, N\right)$, so $\zeta$ comes from there and then $\eta=\delta \zeta=0$. This shows injectivity of the map; surjectivity is left to the reader.

Let us then verify erasability in $M$. Let $M^{\prime}=\operatorname{Ind}_{\mathfrak{g}[[t]] \oplus \mathbb{C} K}^{\hat{\mathfrak{g}}_{\kappa}} M$ and note that restriction of cocycles gives a map of Chevalley complexes

$$
C^{\bullet}\left(\mathfrak{g}((t)), \mathfrak{g} ; M^{\prime}, N\right) \rightarrow C^{\bullet}(\mathfrak{g}[[t]], \mathfrak{g} ; M, N),
$$

which we claim to be a quasi-isomorphism. Granting this for now, we identify the right-hand Chevalley cohomologies above with the Ext groups in the category Rep $G[[t]]$. Indeed, observe that $\operatorname{Rep} G[[t]]$ contains enough injectives: for any representation $N$ of $G$, the space $\Gamma(N)$ of global sections of the vector bundle associated to $N$ over $G[[t]] / G$ is injective. Any representation $V$ of $G[[t]]$ may be embedded into a representation of this type, namely, into $\Gamma(V)$. Clearly, the Chevalley cohomology of $\operatorname{Hom}(M, \Gamma(N))$ coincides with $\operatorname{Ext}_{G[[t]]}(M, \Gamma(N))=\operatorname{Hom}^{G}(M, N)$ by de Rham's theorem on the contractible space $G[[t]] / G$. Thus, we deduce the isomorphism

$$
\mathrm{E}^{k}\left(M^{\prime}, N\right) \simeq \operatorname{Ext}_{G[[t]]}^{k}(M, N) .
$$

The functor $\operatorname{Ext}^{k}$ is left erasable in $\operatorname{Rep} G[[t]]$. Now, if $M$ is already a representation of $\hat{\mathfrak{g}}_{\kappa}$, the inclusion of vector spaces $M \rightarrow M^{\prime}$ that we used above to construct a map (2.2) has a splitting $M^{\prime} \rightarrow M$ which is a homomorphism of $\hat{\mathfrak{g}}_{\kappa}$-modules. This splitting lifts any class $\eta \in \mathrm{E}^{k}(M, N)$ to $\eta^{\prime} \in \mathrm{E}^{k}\left(M^{\prime}, N\right)$. The isomorphic image of $\eta^{\prime}$, via $(2.3)$, in $\operatorname{Ext}_{G[[t]]}^{k}(M, N)$ vanishes after further lifting to $\operatorname{Ext}_{G[[t]]}^{k}\left(M_{1}, N\right)$ via an epimorphism $M_{1} \rightarrow M$, which is an erasure of $\eta$ in $\operatorname{Rep} G[[t]]$ (by the above, we know that such a map $M_{1} \rightarrow M$ exists). We then have a commutative diagram

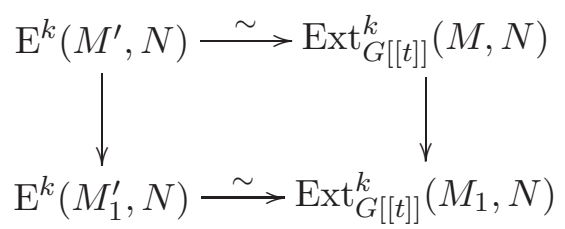

where $M_{1}^{\prime}=\operatorname{Ind}_{\mathfrak{g}[[t]] \oplus \mathbb{C} K}^{\hat{\mathfrak{g}}_{\kappa}} M$, which shows that the image of $\eta^{\prime}$ under the vertical map is zero. Hence the composition $\mathrm{E}^{k}(M, N) \rightarrow \mathrm{E}^{k}\left(M^{\prime}, N\right) \rightarrow \mathrm{E}^{k}\left(M^{\prime}, N\right)$ obtained via the composite epimorphism $M_{1}^{\prime} \rightarrow M^{\prime} \rightarrow M$ sends $\eta$ to zero as well. The epimorphism $M_{1}^{\prime} \rightarrow M$ is then the desired erasure of $\eta$ in $H C\left(\hat{\mathfrak{g}}_{\kappa}, G[[t]]\right)$.

We are left to check that (2.2) is a quasi-isomorphism. Letting

$$
F_{-1} \mathfrak{g}((t))=0, \quad F_{0} \mathfrak{g}((t))=\mathfrak{g}[[t]], \quad F_{1} \mathfrak{g}((t))=\mathfrak{g}((t))
$$

gives increasing filtrations on $\Lambda \mathfrak{g}((t)) / \mathfrak{g}$ and $M^{\prime}$, and hence on their tensor product. (Thus, $F_{0} M^{\prime}=$ $M$ and $F_{1} M^{\prime}=M \oplus\left(t^{-1} \mathfrak{g}\left[t^{-1}\right]\right) \otimes M$.) This leads to a complementary, descending filtration on the Chevalley complex for $(\mathfrak{g}((t)), \mathfrak{g})$ : the maps that vanish on the $p$ th part of the tensor product filtration live in $p$ th degree. The associated graded complex is $C^{\bullet}(\mathfrak{g}[[t]], \mathfrak{g} ; K \otimes M, N)$, where $K$ is the Koszul complex on $\mathfrak{g}((t)) / \mathfrak{g}[[t]]$. Consequently, the restriction (2.2) gives an isomorphism of cohomologies, if we use the Gr of the left-hand Chevalley complex. Because the filtration is Hausdorff and complete on our space of continuous linear maps, cocycles and coboundaries can be lifted order-by-order to the complex from its $\mathrm{Gr}$, leading to an isomorphism of cohomologies in the original map (2.2). ${ }^{2}$

Finally, consider the category $H C\left(\hat{\mathfrak{g}}_{\kappa}, G\right)$ of continuous Harish-Chandra modules for the pair $\left(\hat{\mathfrak{g}}_{\kappa}, G\right)$ : these are the $\mathfrak{g}$-integrable $\hat{\mathfrak{g}}_{\kappa}$-modules (where $\mathfrak{g} \subset \mathfrak{g}_{\kappa}$ is the constant subalgebra) with

${ }^{2}$ This is, of course, the 'collapse of the spectral sequence' argument in [Fuc88, Theorem 1.5.4]. 


\section{E. Frenkel and C. Teleman}

continuous action for the direct limit topology (see Remark 1.1), and on which $K \equiv 1$. Continuity amounts to asking that every vector is annihilated by some subalgebra $t^{N} \mathfrak{g}[[t]]$.

Note that the category $H C\left(\hat{\mathfrak{g}}_{\kappa}, G[[t]]\right)$ is a full subcategory of $H C\left(\hat{\mathfrak{g}}_{\kappa}, G\right)$.

Proposition 2.2. There are natural isomorphisms

$$
\operatorname{Ext}_{H C\left(\hat{\mathfrak{g}}_{\kappa}, G\right)}^{k}\left(\mathbb{V}_{\kappa}, \mathbb{V}_{\kappa}\right) \simeq \operatorname{Ext}_{H C\left(\hat{\mathfrak{g}}_{\kappa}, G[[t]]\right)}^{k}\left(\mathbb{V}_{\kappa}, \mathbb{V}_{\kappa}\right) .
$$

Proof. We shall see below that we have a natural isomorphism

$$
\operatorname{Ext}_{H C(\mathfrak{g}[[t]], G)}^{k}(M, N) \simeq \operatorname{Ext}_{G[[t]]}^{k}(M, N)
$$

for any objects $M, N$ of Rep $G[[t]]$. Assuming this, we can repeat the argument in the proof of Proposition 2.1, with the category $\operatorname{Rep} G[[t]]$ replaced by $H C(\mathfrak{g}[[t]], G)$, to conclude that the Chevalley complex $C^{\bullet}(\mathfrak{g}((t)), \mathfrak{g} ; M, N)$ computes the Ext in $H C\left(\hat{\mathfrak{g}}_{\kappa}, G\right)$.

To verify the isomorphism (2.4), we use the right adjoint functor CoInd to the forgetful functor from $G[[t]]$-modules to $H C(\mathfrak{g}[[t]], G)$. Namely, $\operatorname{CoInd}(N)$ is the space of horizontal sections of the bundle $G[[t]] \times{ }_{G} N$ over $G[[t]] / G$, with flat connection determined from the $\mathfrak{g}[[t]]$-action. It is easy to see that the right derived functors $R^{q}$ CoInd are the de Rham cohomologies with coefficients in the same bundle. We also note that modules of the form $\operatorname{Hom}^{G}(U \mathfrak{g}[[t]] ; N)$ are injective objects in the category $H C\left(\hat{\mathfrak{g}}_{\kappa}, G[[t]]\right)$. Indeed, for any object $M$ of this category $\operatorname{Hom}\left(M, \operatorname{Hom}^{G}(U \mathfrak{g}[[t]] ; N)\right)$ is isomorphic to $\operatorname{Hom}^{G}(M, N)$. The functor CoInd takes $\operatorname{Hom}^{G}(U \mathfrak{g}[[t]] ; N)$ to $\Gamma(N)$. For any object $M$ of $\operatorname{Rep} G[[t]]$, we have therefore a Grothendieck spectral sequence

$$
\operatorname{Ext}_{G[[t]]}^{p}\left(M, R^{q} \operatorname{CoInd}(N)\right) \Rightarrow \operatorname{Ext}_{H C(\mathfrak{g}[t]], G)}^{p+q}(M, N) .
$$

However, if $N$ is already in $\operatorname{Rep} G[[t]], R^{q} \operatorname{CoInd}(N)=0$ for $q>0$ : this is because the flat $N$-bundle over $G[[t]] / G$ becomes isomorphic to the trivial $N$-bundle by a shearing map $(\gamma, n) \mapsto(\gamma, \gamma n)$, and contractibility of the space implies the vanishing of higher cohomology. The spectral sequence degenerates to the desired isomorphism of the Ext.

\section{DG vertex algebra structure and skew-commutativity}

We now wish to compute the algebra $\operatorname{Ext}_{H C\left(\hat{\mathfrak{g}}_{\kappa}, G\right)}^{\bullet}\left(\mathbb{V}_{\kappa}, \mathbb{V}_{\kappa}\right)$. By Proposition 2.1, it is isomorphic to the algebra $H^{\bullet}\left(\mathfrak{g}((t)), \mathfrak{g}\right.$, End $\left.\mathbb{V}_{\kappa}\right)$. Since $\mathbb{V}_{\kappa}$ is an induced module, Shapiro's lemma [Fuc88, Theorem 1.5.4] (see also the end of our proof of Proposition 2.1) implies the following statement.

LEMma 3.1. We have a canonical isomorphism

$$
H^{\bullet}\left(\mathfrak{g}[[t]], \mathfrak{g}, \mathbb{V}_{\kappa}\right) \simeq H^{\bullet}\left(\mathfrak{g}((t)), \mathfrak{g}, \text { End } \mathbb{V}_{\kappa}\right) .
$$

In particular, $H^{\bullet}\left(\mathfrak{g}[[t]], \mathfrak{g}, \mathbb{V}_{\kappa}\right)$ acquires an algebra structure via this isomorphism.

Remark 3.2. A similar argument shows that $H^{\bullet}\left(\mathfrak{g}[[t]], \mathfrak{g}, \mathbb{V}_{\kappa}\right)$ is isomorphic to the relative semiinfinite cohomology $H^{\frac{\infty}{2}+\bullet}\left(\hat{\mathfrak{g}}_{2 \kappa_{\mathrm{c}}}, \mathfrak{g}, \mathbb{V}_{\kappa} \otimes \mathbb{V}_{2 \kappa_{\mathrm{c}}-\kappa}\right)$, which is well defined because $\mathbb{V}_{\kappa} \otimes \mathbb{V}_{2 \kappa_{\mathrm{c}}-\kappa}$ is a module of twice the critical level, as defined in $\S 4$.

Thus, we need to compute the cohomology $H^{\bullet}\left(\mathfrak{g}[[t]], \mathfrak{g}, \mathbb{V}_{\kappa}\right)$. It is realized as the cohomology of the relative Chevalley complex

$$
C^{\bullet}\left(\mathfrak{g}[[t]], \mathfrak{g}, \mathbb{V}_{\kappa}\right)=\left(\mathbb{V}_{\kappa} \otimes \bigwedge^{\bullet}(\mathfrak{g}[[t]] / \mathfrak{g})^{*}\right)^{\mathfrak{g}} .
$$

However, it is not immediately clear what algebra structure is induced on the cohomology of this complex via the isomorphism of Lemma 3.1. We now explain how to define this algebra structure directly on $H^{\bullet}\left(\mathfrak{g}[[t]], \mathfrak{g}, \mathbb{V}_{\kappa}\right)$. 


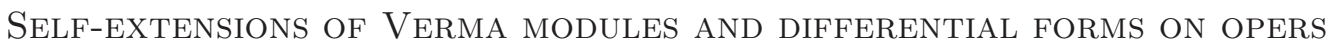

Namely, we will define a DG vertex superalgebra structure on the relative Chevalley complex $C^{\bullet}\left(\mathfrak{g}[[t]], \mathfrak{g}, \mathbb{V}_{\kappa}\right)$. It will induce a vertex superalgebra structure on its cohomology $H^{\bullet}\left(\mathfrak{g}[[t]], \mathfrak{g}, \mathbb{V}_{\kappa}\right)$. We will show that the vertex superalgebra $H^{\bullet}\left(\mathfrak{g}[[t]], \mathfrak{g}, \mathbb{V}_{\kappa}\right)$ is skew-commutative, and hence we will obtain the structure of an ordinary skew-commutative algebra on $H^{\bullet}\left(\mathfrak{g}[[t]], \mathfrak{g}, \mathbb{V}_{\kappa}\right)$. Finally, we will show that the latter coincides with the algebra structure obtained from Lemma 3.1. Hence, by Proposition 2.1, the algebra $H^{\bullet}\left(\mathfrak{g}[[t]], \mathfrak{g}, \mathbb{V}_{\kappa}\right)$ is isomorphic to the algebra $\operatorname{Ext}_{H C\left(\hat{\mathfrak{g}}_{\kappa}, G\right)}\left(\mathbb{V}_{\kappa}, \mathbb{V}_{\kappa}\right)$.

We start by recalling that the $\hat{\mathfrak{g}}_{\kappa}$-module $\mathbb{V}_{\kappa}$ is a vertex algebra (see [FB04, $\left.\S 2.4\right]$ ). Let $\left\{J^{a}\right\}$ be a basis of $\mathfrak{g}$. Denote the element $J^{a} \otimes t^{n} \in \hat{\mathfrak{g}}_{\kappa}$ by $J_{n}^{a}$. Then $\mathbb{V}_{\kappa}$ is freely generated by the vertex operators

$$
Y\left(J_{-1}^{a} v, z\right)=J^{a}(z)=\sum_{n \in \mathbb{Z}} J_{n}^{a} z^{-n-1}
$$

in the sense of the Reconstruction theorem [FB04, §4.4].

Next, recall that any skew-commutative associative superalgebra $V$ with a unit and a superderivation $T$ carries a canonical vertex superalgebra structure with the vertex operators defined by the formula [FB04, $§ 1.4]$

$$
Y(A, z)=\operatorname{mult}\left(e^{z T} A\right)=\sum_{n \geqslant 0} \frac{1}{n !} z^{n} \operatorname{mult}\left(T^{n} A\right) .
$$

Conversely, given a skew-commutative vertex superalgebra $V$, with the vertex operation

$$
Y: V \rightarrow \text { End } V\left[\left[z^{ \pm 1}\right]\right], \quad A \mapsto Y(A, z)=\sum_{n \in \mathbb{Z}} A_{(n)} z^{-n-1}
$$

we recover the skew-commutative algebra product on it by the formula $A, B \mapsto A_{(-1)} B$.

Consider the skew-commutative algebra $\bigwedge^{\bullet}\left(\mathfrak{g}[[t]]^{*}\right)$. It has generators $\psi_{a, n}^{*} \in \mathfrak{g}[[t]]^{*}$, where $a=$ $1, \ldots, \operatorname{dim} \mathfrak{g}, n \leqslant 0$, defined by the formula $\psi_{a, n}^{*}\left(J_{m}^{b}\right)=\delta_{a, b} \delta_{n,-m}$. Define a superderivation $T$ on $\Lambda^{\bullet}\left(\mathfrak{g}[[t]]^{*}\right)$ by the formula $T \cdot \psi_{a, n}^{*}=-(n-1) \psi_{a, n-1}^{*}$. Then $\bigwedge^{\bullet}\left(\mathfrak{g}[[t]]^{*}\right)$ acquires the structure of a vertex superalgebra. It is freely generated by the vertex operators

$$
Y\left(\psi_{a, n}^{*}, z\right)=\psi_{a}^{*}(z)=\sum_{n \leqslant 0} \psi_{a, n}^{*} z^{-n},
$$

in the sense of the Reconstruction theorem [FB04, §4.4].

Let us observe that $\Lambda^{\bullet}\left(\mathfrak{g}[[t]]^{*}\right)$ is a module over the Clifford algebra associated to the vector space $\mathfrak{g}[[t]] \oplus \mathfrak{g}[[t]]^{*}$ with the non-degenerate symmetric bilinear form induced by the residue pairing. This algebra has generators $\psi_{a, n}, \psi_{a, m}^{*}, a=1, \ldots, \operatorname{dim} \mathfrak{g} ; n \geqslant 0, m \leqslant 0$, satisfying the anti-commutation relations

$$
\left[\psi_{a, n}, \psi_{b, m}^{*}\right]_{+}=\delta_{a, b} \delta_{n,-m}, \quad\left[\psi_{a, n}, \psi_{b, m}\right]_{+}=\left[\psi_{a, n}^{*}, \psi_{b, m}^{*}\right]_{+}=0 .
$$

The operators $\psi_{a, m}^{*}$ act on $\Lambda^{\bullet}\left(\mathfrak{g}[[t]]^{*}\right)$ by multiplication and the operators $\psi_{a, n}$ act by contraction. Introduce the generating functions

$$
\psi_{a}(z)=\sum_{n \geqslant 0} \psi_{a, n} z^{-n-1}
$$

We consider the tensor product vertex superalgebra structure on the Chevalley complex

$$
C^{\bullet}\left(\mathfrak{g}[[t]], \mathbb{V}_{\kappa}\right)=\mathbb{V}_{\kappa} \otimes \bigwedge^{\bullet}\left(\mathfrak{g}[[t]]^{*}\right) .
$$

For $A \in C^{\bullet}\left(\mathfrak{g}[[t]], \mathbb{V}_{\kappa}\right)$ we denote by $p(A)$ its parity. The vertex operation

$$
Y: C^{\bullet}\left(\mathfrak{g}[[t]], \mathbb{V}_{\kappa}\right) \rightarrow \text { End } C^{\bullet}\left(\mathfrak{g}[[t]], \mathbb{V}_{\kappa}\right)\left[\left[z^{ \pm 1}\right]\right]
$$




\section{E. Frenkel and C. Teleman}

may be determined by linearity by the explicit formula

$$
\begin{aligned}
& Y\left(J_{n_{1}}^{a_{1}} \cdots J_{n_{k}}^{a_{k}} v \otimes \psi_{b_{1}, m_{1}}^{*} \cdots \psi_{b_{l}, m_{l}}^{*}, z\right) \\
& \quad=\prod_{i=1}^{k} \frac{1}{\left(-n_{i}-1\right) !} \prod_{j=1}^{l} \frac{1}{\left(-m_{j}\right) !}: \partial_{z}^{-n_{1}-1} J^{a_{1}}(z) \cdots \partial_{z}^{-n_{k}-1} J^{a_{k}}(z): \partial_{z}^{-m_{1}} \psi_{b_{1}}^{*}(z) \cdots \partial_{z}^{-m_{l}} \psi_{b_{l}}^{*}(z),
\end{aligned}
$$

where the colons denote normal ordering.

The differential $d$ of Lie algebra cohomology is given by the formula

$$
d=\operatorname{Res}_{z=0}\left(\sum_{a} J^{a}(z) \psi_{a}^{*}(z)-\frac{1}{2} \sum_{a, b, c} \mu_{c}^{a b} \psi_{a}^{*}(z) \psi_{b}^{*}(z) \psi_{c}(z)\right) d z,
$$

where $\left(\mu_{c}^{a b}\right)$ are the structure constants of $\mathfrak{g}$ :

$$
\left[J^{a}, J^{b}\right]=\sum_{c} \mu_{c}^{a b} J^{c}
$$

One checks easily that $d$ is a superderivation of the vertex algebra $C^{\bullet}\left(\mathfrak{g}[[t]], \mathbb{V}_{\kappa}\right)$, i.e. we have

$$
Y(d A, z)=[d, Y(A, z)]_{ \pm},
$$

where the sign of the commutator depends on whether the parity of $A \in C^{\bullet}\left(\mathfrak{g}[[t]], \mathbb{V}_{\kappa}\right)$ is even or odd. Therefore the differential $d$ gives $C^{\bullet}\left(\mathfrak{g}[[t]], \mathbb{V}_{\kappa}\right)$ the structure of a DG vertex superalgebra. Thus, its cohomology is a graded vertex superalgebra.

Next, observe that the relative Chevalley complex $C^{\bullet}\left(\mathfrak{g}[[t]], \mathfrak{g}, \mathbb{V}_{\kappa}\right)$ is equal to the intersection of the kernels of the operators $\psi_{i, 0}=\operatorname{Res}_{z=0} \psi_{i}(z) d z$ and $\widehat{J}_{0}^{a}=\operatorname{Res}_{z=0} \widehat{J}^{a}(z) d z, a=1, \ldots, \operatorname{dim} \mathfrak{g}$, where

$$
\widehat{J}^{a}(z)=J^{a}(z)-\sum_{b} \mu_{c}^{a b} \psi_{b}^{*}(z) \psi_{c}(z)
$$

These operators are superderivations of the vertex algebra structure on $C^{\bullet}\left(\mathfrak{g}[[t]], \mathbb{V}_{\kappa}\right)$, i.e. they satisfy relations similar to (3.3). Therefore the intersection of the kernels of these operators is a vertex subalgebra of $C^{\bullet}\left(\mathfrak{g}[[t]], \mathbb{V}_{\kappa}\right)$. Hence we obtain that $C^{\bullet}\left(\mathfrak{g}[[t]], \mathfrak{g}, \mathbb{V}_{\kappa}\right)$ also carries the structure of a DG vertex superalgebra, and so its cohomology $H^{\bullet}\left(\mathfrak{g}[[t]], \mathfrak{g}, \mathbb{V}_{\kappa}\right)$ is a graded vertex superalgebra.

The following result (and its generalization described in Remark 3.5) has been suggested to us by Gaitsgory.

Proposition 3.3. The vertex superalgebras $C^{\bullet}\left(\mathfrak{g}[[t]], \mathbb{V}_{\kappa}\right)$ and $C^{\bullet}\left(\mathfrak{g}[[t]], \mathfrak{g}, \mathbb{V}_{\kappa}\right)$ are homotopy skewcommutative. Thus, their cohomology vertex superalgebras $H^{\bullet}\left(\mathfrak{g}[[t]], \mathbb{V}_{\kappa}\right)$ and $H^{\bullet}\left(\mathfrak{g}[[t]], \mathfrak{g}, \mathbb{V}_{\kappa}\right)$ are skew-commutative.

Proof. Suppose that $V$ is a vertex superalgebra with the vertex operation $Y$ (see (3.1)). Restricting it to the negative powers of $z$ we obtain an operation $Y_{-}: V \rightarrow$ End $V \otimes z^{-1} \mathbb{C}\left[\left[z^{-1}\right]\right]$, which gives $V$ the structure of a vertex Lie superalgebra (see $[\mathrm{FB} 04, \S 16.1]$ ). Recall that a vertex superalgebra is called skew-commutative if $Y_{-} \equiv 0$.

We will introduce the following notation for $A, B \in V$ :

$$
\begin{array}{ll}
Y_{(m)}(A, B)=A_{(m)} B, & m \geqslant 0, \\
Y_{(m)}(A, B)=0, & m<0 .
\end{array}
$$

Then the vertex Lie superalgebra operation $Y_{-}$on $V$ is encoded by the linear maps $Y_{(m)}: V \otimes V \rightarrow$ $V$. Thus, $V$ is skew-commutative if and only if $Y_{(m)}=0$ for all $m \geqslant 0$. 


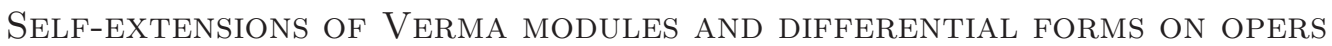

We recall that the operations $Y_{(m)}$ satisfy the following identities:

$$
\begin{aligned}
Y_{(m)}(A, B)= & (-1)^{p(A) p(B)} \sum_{n=0}^{m} \frac{1}{n !}(-1)^{m-n} T^{n} Y_{(m-n)}(B, A), \\
Y_{(m)}(T A, B)= & Y_{(m-1)}(A, B), \\
Y_{(m)}\left(A, B_{(-1)} C\right)= & Y_{(m)}(A, B)_{(-1)} C+(-1)^{p(A) p(B)} B_{(-1)} Y_{(m)}(A, C) \\
& +\sum_{j=0}^{m-1} Y_{(m-j-1)}\left(Y_{(j)}(A, B), C\right)
\end{aligned}
$$

(see $[\mathrm{FB} 04, \S 16.1])$.

Now consider the DG vertex superalgebra $C^{\bullet}=C^{\bullet}\left(\mathfrak{g}[[t]], \mathbb{V}_{\kappa}\right)$ with the differential $d$. Formula (3.3) implies the following identity:

$$
d Y_{(m)}(A, B)=Y_{(m)}(d A, B)+(-1)^{p(A)} Y_{(m)}(A, d B) .
$$

We will show that $C^{\bullet}$ is homotopy skew-commutative, i.e. we will construct bilinear maps

$$
Z_{(m)}: C^{\bullet} \otimes C^{\bullet} \rightarrow C^{\bullet}, \quad m \geqslant 0,
$$

of cohomological degree -1 such that

$$
d Z_{(m)}(A, B)-Z_{(m)}(d A, B)+(-1)^{p(A)} Z_{(m)}(A, d B)=Y_{(m)}(A, B) .
$$

This will imply that the cohomology vertex superalgebra $H^{\bullet}\left(\mathfrak{g}[[t]], \mathbb{V}_{\kappa}\right)$ is skew-commutative.

In order to construct it, we need to formulate some general properties of biderivations of vertex Lie algebras. Let $V$ be a vertex Lie algebra. We will call a collection of linear maps $Z_{(m)}: V \otimes V \rightarrow$ $V, m \geqslant 0$, a skew-symmetric biderivation if they satisfy the following properties:

$$
\begin{aligned}
Z_{(m)}(A, B)= & (-1)^{p(A) p(B)} \sum_{n=0}^{m} \frac{1}{n !}(-1)^{m-n} T^{n} Z_{(m-n)}(B, A), \\
Z_{(m)}(T A, B)= & Z_{(m-1)}(A, B), \\
Z_{(m)}\left(A, B_{(-1)} C\right)= & Z_{(m)}(A, B)_{(-1)} C+(-1)^{p(A) p(B)} B_{(-1)} Z_{(m)}(A, C) \\
& +\sum_{j=0}^{m-1} Y_{(m-j-1)}\left(Z_{(j)}(A, B), C\right) .
\end{aligned}
$$

In particular, $\left(Y_{(m)}\right)$ is a skew-symmetric biderivation.

The following lemma, which is proved by a straightforward calculation using the identities (3.4)-(3.6), (3.9)-(3.11) and (3.7), is a generalization of the well-known properties of the ordinary derivations.

LEMMA 3.4.

(1) Let $V$ be a vertex superalgebra freely generated by elements $A_{i}, i \in I$. Suppose that we are given $Z_{(m)}\left(A_{i}, A_{j}\right) \in V$ for all $m \geqslant 0$ and $i, j \in I$, satisfying the identities (3.9). Then this assignment may be extended uniquely to a skew-symmetric biderivation of $V$.

(2) Let $V$ be a $D G$ vertex superalgebra, freely generated by $A_{i}, i \in I$, and $\left(Z_{(m)}\right)$ a skew-symmetric biderivation of $V$. Suppose that $\left(Z_{(m)}\right)$ satisfies the identity (3.8) specialized to $A=A_{i}$, $B=A_{j}, i, j \in I$. Then $\left(Z_{(m)}\right)$ satisfies the identity (3.8) for all $A, B \in V$ and hence gives $V$ the structure of a homotopy skew-commutative $D G$ vertex superalgebra.

Recall that our DG vertex superalgebra $C^{\bullet}$ is generated by $J_{-1}^{a} v$ and $\psi_{a, 0}^{*}, a=1, \ldots, \operatorname{dim} \mathfrak{g}$. In light of Lemma 3.4, in order to construct a homotopy $\left(Z_{(m)}\right)$ on $C^{\bullet}$, it is sufficient to define 


\section{E. Frenkel and C. Teleman}

the $Z_{(m)}$ on these generators in such a way that they satisfy (3.8) and (3.9). We define them by the following formulas:

$$
\begin{aligned}
Z_{(m)}\left(J_{-1}^{a} v, \psi_{b, 0}^{*}\right) & =-Z_{(m)}\left(\psi_{b, 0}^{*}, J_{-1}^{a} v\right)=\frac{1}{2} \delta_{a, b} \delta_{m, 0}, \\
Z_{(m)}\left(J_{-1}^{a} v, J_{-1}^{b} v\right) & =Z_{(m)}\left(\psi_{a, 0}^{*}, \psi_{b, 0}^{*}\right)=0 .
\end{aligned}
$$

One checks by a direct calculation that these formulas satisfy the identities (3.8) and (3.9). Hence they can be extended uniquely to a skew-symmetric biderivation of $C^{\bullet}$ which gives rise to the structure of a skew-commutative homotopy DG superalgebra on it. This proves the statement of the proposition for the Chevalley complex $C^{\bullet}=C^{\bullet}\left(\mathfrak{g}[[t]], \mathbb{V}_{\kappa}\right)$. In order to prove the statement for the relative Chevalley complex $C^{\bullet}\left(\mathfrak{g}[[t]], \mathfrak{g}, \mathbb{V}_{\kappa}\right)$, which is a DG vertex subalgebra of $C^{\bullet}$, it is sufficient to show that our biderivation $\left(Z_{(m)}\right)$ preserves this subcomplex. But it follows from the construction that the maps $Z_{(m)}$ commute with the action of $\psi_{a, 0}^{*}$ and $\widehat{J}_{0}^{a}$ on $C^{\bullet}$. Hence the homotopy $\left(Z_{(m)}\right)$ preserves $C^{\bullet}\left(\mathfrak{g}[[t]], \mathfrak{g}, \mathbb{V}_{\kappa}\right)$. This completes the proof.

Remark 3.5. The vertex algebra $\mathbb{V}_{\kappa}$ is the enveloping vertex algebra of the vertex Lie algebra associated to the affine Kac-Moody algebra $\hat{\mathfrak{g}}_{\kappa}$. An analogue of the complex $C^{\bullet}\left(\mathfrak{g}[[t]], \mathbb{V}_{\kappa}\right)$ may be defined for the enveloping vertex algebra of any vertex Lie algebra. This cohomology complex is then a DG vertex superalgebra. The above proof carries over verbatim to this more general context, and we obtain that this cohomology complex is always homotopy skew-commutative.

Thus, we obtain that $H^{\bullet}\left(\mathfrak{g}[[t]], \mathfrak{g}, \mathbb{V}_{\kappa}\right)$ is a skew-commutative vertex superalgebra. Therefore the bilinear operation on $H^{\bullet}\left(\mathfrak{g}[[t]], \mathfrak{g}, \mathbb{V}_{\kappa}\right)$ given by the formula

$$
A, B \mapsto A_{(-1)} B
$$

defines the structure of a skew-commutative associative algebra on it.

For a general vertex algebra this operation is non-commutative and non-associative, so it does not give it the structure of an algebra. But in our case, according to Proposition 3.3, it gives $H^{\bullet}\left(\mathfrak{g}[[t]], \mathfrak{g}, \mathbb{V}_{\kappa}\right)$ the structure of a skew-commutative associative algebra. In the next lemma we show that the above product coincides with the product structure on the cohomology $H^{\bullet}\left(\mathfrak{g}[[t]], \mathfrak{g}, \mathbb{V}_{\kappa}\right)$ induced by its isomorphism with the algebra of the Ext from Lemma 3.1. Thus, we obtain that the latter is skew-commutative, which is not obvious otherwise.

Let us observe that the Fourier coefficients of the series (3.2) may be viewed as elements of the Chevalley complex $C^{\bullet}\left(\mathfrak{g}((t)), \mathfrak{g}\right.$, End $\left.\mathbb{V}_{\kappa}\right)$. Indeed, the $l$ th group of this complex is spanned by skew-symmetric continuous $l$-linear maps from $\mathfrak{g}((t))$ to End $\mathbb{V}_{\kappa}$. To a Fourier coefficient $A_{(n)}=$ $\int Y(A, z) z^{n} d z$ we assign the $l$-linear functional whose value on $J_{p_{1}}^{b_{1}} \wedge \cdots \wedge J_{p_{l}}^{b_{l}}$ is the endomorphism of $\mathbb{V}_{\kappa}$ that equals the coefficient in front of $\psi_{b_{1}, p_{1}}^{*} \cdots \psi_{b_{l}, p_{l}}^{*}$ in $A_{(n)}$.

LEMMA 3.6. The isomorphism

$$
H^{\bullet}\left(\mathfrak{g}[[t]], \mathfrak{g}, \mathbb{V}_{\kappa}\right) \simeq H^{\bullet}\left(\mathfrak{g}((t)), \mathfrak{g}, \operatorname{End}_{\mathbb{C}} \mathbb{V}_{\kappa}\right)
$$

of Lemma 3.1 may be realized as follows. Given a cohomology class in $H^{\bullet}\left(\mathfrak{g}[[t]], \mathfrak{g}, \mathbb{V}_{\kappa}\right)$, we represent it by a cocycle $A$ in $C^{\bullet}\left(\mathfrak{g}[[t]], \mathfrak{g}, \mathbb{V}_{\kappa}\right)$ and associate to it the cohomology class of $A_{(-1)}$ which is a cocycle in $C^{\bullet}\left(\mathfrak{g}((t)), \mathfrak{g}\right.$, End $\left.\mathbb{V}_{\kappa}\right)$. Then the natural product structure on $H^{\bullet}\left(\mathfrak{g}((t)), \mathfrak{g}\right.$, End $\left.\mathbb{V}_{\kappa}\right)$ induces a product structure on $H^{\bullet}\left(\mathfrak{g}[[t]], \mathfrak{g}, \mathbb{V}_{\kappa}\right)$ which is given by formula (3.12). In particular, the latter is automatically associative.

Proof. Formula (3.3) implies that

$$
\left[d, A_{(-1)}\right]_{ \pm}=(d A)_{(-1)}, \quad A \in C^{\bullet}\left(\mathfrak{g}[[t]], \mathfrak{g}, \mathbb{V}_{\kappa}\right) .
$$

Therefore if $A$ is a cocycle (respectively, a coboundary) in $C^{\bullet}\left(\mathfrak{g}[[t]], \mathfrak{g}, \mathbb{V}_{\kappa}\right)$, then $A_{(-1)}$ is a cocycle (respectively, a coboundary) in $C^{\bullet}\left(\mathfrak{g}((t)), \mathfrak{g}\right.$, End $\left.\mathbb{V}_{\kappa}\right)$. Thus, we obtain a well-defined map 


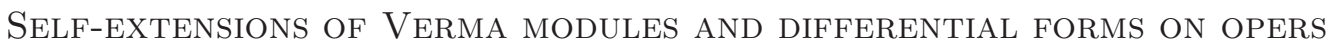

from $H^{\bullet}\left(\mathfrak{g}[[t]], \mathfrak{g}, \mathbb{V}_{\kappa}\right)$ to $H^{\bullet}\left(\mathfrak{g}((t)), \mathfrak{g}\right.$, End $\left.\mathbb{V}_{\kappa}\right)$. Let us show that this map coincides with the isomorphism (3.13) obtained via the Shapiro lemma.

Recall the defining property of the isomorphism (3.13). If $\omega$ is any cocycle in the Chevalley complex representing a cohomology class $\bar{\omega}$ in $H^{\bullet}\left(\mathfrak{g}((t)), \mathfrak{g}\right.$, End $\left.\mathbb{V}_{\kappa}\right)$, then the cohomology class in $H^{\bullet}\left(\mathfrak{g}[[t]], \mathfrak{g}, \mathbb{V}_{\kappa}\right)$ corresponding to $\bar{\omega}$ under the isomorphism (3.13) is obtained by restricting $\omega$ to $\Lambda^{\bullet}(\mathfrak{g}[[t]])$ and applying the corresponding endomorphism of $\mathbb{V}_{\kappa}$ to the vacuum vector $\mathbb{V}_{\kappa}$. Recall the vacuum axiom of the vertex algebra: in any vertex algebra $V$ with the vacuum vector $|0\rangle$ we have $A_{(-1)}|0\rangle=A$ for any $A$. It follows that if $\omega=A_{(-1)}$, then the result of the above procedure will be $A$.

This proves that the isomorphism (3.13) is indeed realized by the assignment $A \mapsto A_{(-1)}$. But the product of $A_{(-1)}$ and $B_{(-1)}$ in $H^{\bullet}\left(\mathfrak{g}((t)), \mathfrak{g}, \operatorname{End}_{\mathbb{C}} \mathbb{V}_{\kappa}\right)$ is just the composition $A_{(-1)} \circ B_{(-1)}$. This induces the following product structure on $H^{\bullet}\left(\mathfrak{g}[[t]], \mathfrak{g}, \mathbb{V}_{\kappa}\right): A, B \mapsto\left(A_{(-1)} \circ B_{(-1)}\right)|0\rangle=A_{(-1)} B$, i.e. the one given by formula (3.12). This completes the proof.

\section{Cohomology of the vacuum module of critical level}

Now we specialize to the critical inner product $\kappa_{\mathrm{c}}=-\frac{1}{2} \kappa_{\mathrm{K}}$, where $\kappa_{\mathrm{K}}$ denotes the Killing form on $\mathfrak{g}$. Thus, by definition,

$$
\kappa_{\mathrm{c}}(x, y)=-\frac{1}{2} \operatorname{Tr}(\operatorname{ad} x \operatorname{ad} y) .
$$

In order to simplify notation, we will denote $\mathbb{V}_{\kappa_{\mathrm{c}}}$ by $\mathbb{V}$.

In cohomological degree zero we have

$$
H^{0}(\mathfrak{g}[[t]], \mathfrak{g}, \mathbb{V})=\mathbb{V}^{\mathfrak{g}[[t]]} \simeq \operatorname{End}_{\hat{\mathfrak{g}}_{\kappa_{\mathrm{c}}}} \mathbb{V}
$$

This algebra has been described in [FF92] and [Fre04]. Let us recall this result.

First we need to define $\mathfrak{g}$-opers (see [DS85, BD05, Fre04]). Choose a Cartan decomposition $\mathfrak{g}=\mathfrak{n}_{-} \oplus \mathfrak{h} \oplus \mathfrak{n}$ and Chevalley generators $f_{i}, i=1, \ldots, \ell$, of $\mathfrak{n}_{-}$. Denote by $\mathfrak{b}$ the direct sum $\mathfrak{h} \oplus \mathfrak{n}$ and by $N$ the Lie group of $\mathfrak{n}$. Then by definition a $\mathfrak{g}$-oper on the disc $D=\operatorname{Spec} \mathbb{C}[[t]]$ is an equivalence class of first-order operators

$$
\nabla=\partial_{t}+\sum_{i=1}^{\ell} f_{i}+\mathbf{v}(t), \quad \mathbf{v}(t) \in \mathfrak{b}[[t]],
$$

with respect to the gauge action of the group $N[[t]]$ given by the formula

$$
g \cdot\left(\partial_{t}+A(t)\right)=\partial_{t}+g A(t) g^{-1}-\partial_{t} g \cdot g^{-1} .
$$

We denote the space of $\mathfrak{g}$-opers on $D$ by $\operatorname{Op}_{\mathfrak{g}}(D)$.

Let us include the element $p_{-1}=\sum_{i=1}^{\ell} f_{i}$ into a principal $\mathfrak{s l}_{2}$-triple $\left\{p_{-1}, 2 \check{\rho}, p_{1}\right\}$, where $\check{\rho} \in \mathfrak{h}$ is the sum of the fundamental coweights. The space of invariants in $\mathfrak{n}$ of the adjoint action of $p_{1}$ has a basis $p_{j}, j=1, \ldots, \ell$, of elements such that $\left[\check{\rho}, p_{j}\right]=d_{j} p_{j}$, where $\left\{d_{1}, \ldots, d_{\ell}\right\}$ is the set of exponents of $\mathfrak{g}$.

It is known from [DS85] that the above action of $N[[t]]$ is free and each equivalence class contains a unique operator of the form $\partial_{t}+\sum_{i=1}^{\ell} f_{i}+\mathbf{v}(t)$, where

$$
\mathbf{v}(t)=\sum_{j=1}^{\ell} v_{j}(t) \cdot p_{j}, \quad v_{j}(t) \in \mathbb{C}[[t]] .
$$




\section{E. Frenkel and C. Teleman}

The series $v_{1}(t)$ transforms as a projective connection, while $v_{j}(t), j>1$, transforms as a $\left(d_{j}+1\right)$ differential (see e.g. [Fre04]). Thus, we obtain an isomorphism

$$
\mathrm{Op}_{\mathfrak{g}}(D) \simeq \operatorname{Proj}(D) \times \bigoplus_{j=2}^{\ell} \Gamma\left(D, \omega^{\otimes\left(d_{j}+1\right)}\right) .
$$

This isomorphism is coordinate-independent, but it depends in the obvious way on the choice of the $f_{i}$ and the $p_{j}$. The space $\operatorname{Proj}(D)$ is an affine space modeled on the space $\Gamma\left(D, \omega^{\otimes 2}\right)$ of quadratic differentials on $D$. It follows from the identification (4.2) that the algebra $\operatorname{Fun}\left(\mathrm{Op}_{L_{\mathfrak{g}}}(D)\right)$ has a natural filtration, and the associated graded algebra is canonically isomorphic to $\operatorname{Fun}\left(C_{\mathfrak{g}^{*}, \omega}\right)$ (see [BD05, §§ 3.1.12-3.1.14], for more details).

The module $\mathbb{V}$ has a PBW filtration, and the associated graded gr $\mathbb{V}$ is isomorphic to $\mathbb{V}^{\text {cl }}$. This induces a filtration on $\mathbb{V}^{\mathfrak{g}[[t]]}=\operatorname{End}_{\hat{\mathfrak{g}}_{\kappa_{\mathrm{c}}}} \mathbb{V}$ and we obtain a natural homomorphism of algebras

$$
\operatorname{gr} \operatorname{End}_{\hat{\mathfrak{g}}_{\kappa_{\mathrm{c}}}} \mathbb{V} \rightarrow\left(\mathbb{V}^{\mathrm{cl}}\right)^{\mathfrak{g}[[t]]}=\operatorname{Fun}\left(C_{\mathfrak{g}^{*}, \omega}\right) .
$$

Let ${ }^{L} \mathfrak{g}$ be the Lie algebra that is Langlands dual to $\mathfrak{g}$, so that its Cartan matrix is the transpose to that of $\mathfrak{g}$.

Theorem 4.1 [FF92, Fre04]. There is a canonical isomorphism of filtered algebras

$$
\operatorname{End}_{\hat{\mathfrak{g}}_{\kappa_{c}}} \mathbb{V} \simeq \operatorname{Fun}\left(\mathrm{Op}_{L_{\mathfrak{g}}}(D)\right)
$$

such that the following diagram is commutative.

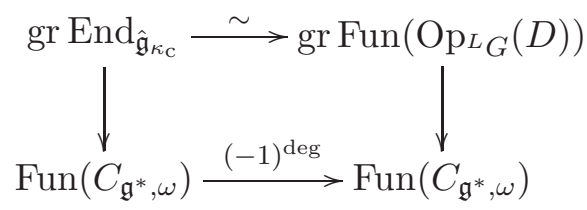

(Here $(-1)^{\operatorname{deg}}$ is the automorphism taking value $(-1)^{n}$ on the subspace of elements of degree $n$.)

Next, we construct maps $H^{i}(\mathfrak{g}[[t]], \mathfrak{g}, \mathbb{V}) \rightarrow H^{i+1}(\mathfrak{g}[[t]], \mathfrak{g}, \mathbb{V})$ similarly to the classical case, by deforming the critical inner product. Consider the one-parameter family of vacuum modules

$$
\mathbb{V}_{\kappa}=\operatorname{Ind}_{\mathfrak{g}[[t]] \oplus \mathbb{C} K}^{\hat{\mathfrak{g}}_{\kappa}} \mathbb{C}
$$

where $\kappa=\kappa_{\mathrm{c}}+h \kappa_{0}$, with respect to the parameter $h$. This family gives rise to a one-parameter family of Chevalley complexes $C^{\bullet}\left(\mathfrak{g}[[t]], \mathfrak{g}, \mathbb{V}_{\kappa}\right)$.

Proposition 4.2. For $\kappa \neq \kappa_{\mathrm{c}}$ the cohomology $H^{i}\left(\mathfrak{g}[[t]], \mathfrak{g}, \mathbb{V}_{\kappa}\right)$ vanishes for $i>0$, and $H^{0}$ $\left(\mathfrak{g}[[t]], \mathfrak{g}, \mathbb{V}_{\kappa}\right)=\mathbb{C}$.

Proof. Consider the contragredient module $\mathbb{V}_{\kappa}^{\vee}$. It follows from the results of [KK79] that for generic $\kappa$ the $\hat{\mathfrak{g}}_{\kappa}$-module $\mathbb{V}_{\kappa}$ is irreducible. Therefore the natural homomorphism of $\hat{\mathfrak{g}}_{\kappa}$-modules $\mathbb{V}_{\kappa} \rightarrow \mathbb{V}_{\kappa}^{\vee}$ is an isomorphism. It follows that $\mathbb{V}_{\kappa}$ is $\mathfrak{g} \otimes t \mathbb{C}[[t]]$-cofree, and we obtain the assertion of the proposition using Shapiro's lemma. A proof that works for an arbitrary $\kappa \neq \kappa_{\mathrm{c}}$ (when $\mathbb{V}_{\kappa}$ may be reducible) will be given after the proof of Theorem 4.4.

Now, in the same way as in the classical case, we obtain linear maps

$$
\varphi_{\kappa_{0}}^{i}: H^{i}(\mathfrak{g}[[t]], \mathfrak{g}, \mathbb{V}) \rightarrow H^{i+1}(\mathfrak{g}[[t]], \mathfrak{g}, \mathbb{V}) .
$$

In particular, for $i=0$ we obtain a map

$$
\varphi_{\kappa_{0}}=\varphi_{\kappa_{0}}^{0}: \operatorname{Fun}\left(\mathrm{Op}_{L_{\mathfrak{g}}}(D)\right) \rightarrow H^{1}(\mathfrak{g}[[t]], \mathfrak{g}, \mathbb{V}) .
$$




\section{SELF-EXTENSIONS OF VERMa MOdUles AND DifFERENTIAL FORMS ON OPERS}

Explicitly, this map looks as follows. Suppose that $P \in \operatorname{Fun}\left(\mathrm{Op}_{L}(D)\right) \simeq \mathbb{V}_{\mathfrak{g}}[[t]]$ and let us compute the corresponding one-cocycle in the Chevalley complex $C^{\bullet}(\mathfrak{g}[[t]], \mathfrak{g}, \mathbb{V})$, which is a linear map $\varphi_{\kappa_{0}}(P): \mathfrak{g} \otimes t \mathbb{C}[[t]] \rightarrow \mathbb{V}$. It follows from the definition that

$$
\left.x(t) \mapsto \frac{1}{h} x(t) \cdot \widetilde{P}\right|_{h=0},
$$

where $\widetilde{P}$ is an arbitrary lifting of $P$ to $\mathbb{V}_{\kappa}$ considered as a free module over $\mathbb{C}[h]$.

Lemma 4.3. The maps $\varphi_{\kappa_{0}}^{i}$ satisfy the Leibniz rule with respect to the natural action of Fun $\left(\mathrm{Op}_{L_{\mathfrak{g}}}(D)\right) \simeq \operatorname{End}_{\hat{\mathfrak{g}}_{\kappa_{\mathrm{c}}}} \mathbb{V}$ on $H^{1}(\mathfrak{g}[[t]], \mathfrak{g}, \mathbb{V})$. In particular, $\varphi_{\kappa_{0}}^{0}$ necessarily factors through the de Rham differential

$$
d: \operatorname{Fun}\left(\mathrm{Op}_{L_{\mathfrak{g}}}(D)\right) \rightarrow \Omega^{1}\left(\mathrm{Op}_{L_{\mathfrak{g}}}(D)\right)
$$

We also have $\varphi_{\kappa_{0}}^{i+1} \circ \varphi_{\kappa_{0}}^{i}=0$ for all $i \geqslant 0$.

Proof. After trivializing the terms $C^{i}\left(\mathfrak{g}[[t]], \mathfrak{g}, \mathbb{V}_{\kappa}\right)$ of the Chevalley complex as bundles over Spec $\mathbb{C}[h]$, we can expand the differential $\delta_{h}$ in powers of $h$ :

$$
\delta_{h}=\delta_{0}+h \delta_{1}+h^{2} \delta_{2}+\cdots .
$$

Note that $\delta_{0}$ is the differential on $C^{i}(\mathfrak{g}[[t]], \mathfrak{g}, \mathbb{V})$, and $\delta_{1}=\varphi_{\kappa_{0}}$ (here we omit the upper index $i$ to simplify notation). From $\delta_{h}^{2}=0$, we find that $\delta_{0}^{2}=0$ (which is clear anyway, as $\delta_{0}$ is a differential); additionally, with $[,]_{+}$denoting the supercommutator,

$$
\left[\delta_{0}, \delta_{1}\right]_{+}=0 \text { and } \delta_{1}^{2}=-\left[\delta_{0}, \delta_{2}\right]_{+} .
$$

The first equality implies that $\delta_{1}=\varphi_{\kappa_{0}}$ maps $\delta_{0}$-cocycles to $\delta_{0}$-cocycles and so indeed gives rise to a well-defined map $H^{i}(\mathfrak{g}[[t]], \mathfrak{g}, \mathbb{V}) \rightarrow H^{i+1}(\mathfrak{g}[[t]], \mathfrak{g}, \mathbb{V})$, while the second equality means that at the level of cohomologies we have $\delta_{1}^{2}=0$.

To see that the Leibniz rule holds, observe that $\delta_{h}$ preserves the multiplicative structure on the Chevalley complex computing

$$
H^{\bullet}\left(\mathfrak{g}((t)), \mathfrak{g}, \text { End } \mathbb{V}_{\kappa}\right) \simeq H^{\bullet}\left(\mathfrak{g}[[t]], \mathfrak{g}, \mathbb{V}_{\kappa}\right) .
$$

Thus we have

$$
\delta_{h}(\underset{h}{*} B)=\left(\delta_{h} A\right) \underset{h}{*} B+(-1)^{\operatorname{deg} A} A \underset{h}{*}\left(\delta_{h} B\right),
$$

where ${ }_{h}^{*}$ denotes the product on the complex depending on the parameter $h$. Expanding $\delta_{h}, A$ and $B$ in powers of $h$ and using the fact that $\delta_{0}$ preserves the multiplicative structure on the Chevalley complex at $h=0$, we obtain that

$$
\delta_{1}\left(A_{0} * B_{0}\right)=\left(\delta_{1} A_{0}\right) * B+(-1)^{\operatorname{deg} A} A *\left(\delta_{1} B\right) .
$$

In particular, if we choose $A_{0}$ to be an element of $H^{0}(\mathfrak{g}((t)), \mathfrak{g}$, End $\mathbb{V})=\operatorname{Fun}\left(\mathrm{Op}_{L_{\mathfrak{g}}}(D)\right)$, we obtain the assertion of the lemma.

Since $\mathrm{Op}_{L_{\mathfrak{g}}}(D)$ is an affine space modeled on the vector space $C_{\mathfrak{g}^{*}, \omega}$, the map $\Omega^{1}\left(\mathrm{Op}_{L_{\mathfrak{g}}}(D)\right) \rightarrow$ $H^{1}(\mathfrak{g}[[t]], \mathfrak{g}, \mathbb{V})$ gives rise to a map

$$
C_{\mathfrak{g}^{*}, \omega}^{*} \rightarrow H^{1}(\mathfrak{g}[[t]], \mathfrak{g}, \mathbb{V}) .
$$

If we multiply $\kappa_{0}$ by $\lambda$, then the map (4.4) will get divided by $\lambda$, as in the classical case.

Now we are ready to describe the cohomology algebra $H^{\bullet}(\mathfrak{g}[[t]], \mathfrak{g}, \mathbb{V})$. The following assertion means that this algebra is a skew-commutative polynomial algebra with the even generators being the generators of the polynomial algebra $\operatorname{Fun}\left(\mathrm{Op}_{L} G(D)\right)$ and the odd generators being the images of some basis elements of $C_{\mathfrak{g}^{*}, \omega}^{*}$ under the map (4.4) which turns out to be injective. 


\section{E. Frenkel and C. Teleman}

TheOREM 4.4. For each non-zero invariant inner product $\kappa_{0}$ on $\mathfrak{g}$ we have an isomorphism

$$
H^{\bullet}(\mathfrak{g}[[t]], \mathfrak{g}, \mathbb{V}) \simeq \Omega^{\bullet}\left(\mathrm{Op}_{L_{\mathfrak{g}}}(D)\right)=\operatorname{Fun}\left(\mathrm{Op}_{L_{\mathfrak{g}}}(D)\right) \otimes \bigwedge^{\bullet}\left(C_{\mathfrak{g}^{*}, \omega}^{*}\right),
$$

where $\Omega^{\bullet}\left(\mathrm{Op}_{L_{\mathfrak{g}}}(D)\right)$ is the algebra of differential forms on $\mathrm{Op}_{L_{\mathfrak{g}}}(D)$, such that the map $\varphi_{\kappa_{0}}$ gets identified with the de Rham differential on $\Omega^{\bullet}\left(\mathrm{Op}_{L_{\mathfrak{g}}}(D)\right)$. If we rescale $\kappa_{0}$ by $\lambda$, then this isomorphism gets rescaled by $\lambda^{-i}$ on the $i$ th cohomology.

Proof. Let us describe the strategy of the proof. We will compute the cohomology $H^{\bullet}(\mathfrak{g}[[t]], \mathfrak{g}, \mathbb{V})$ using the spectral sequence induced by the PBW filtration on $\mathbb{V}$. The first term of this spectral sequence is equal to the cohomology $H^{\bullet}\left(\mathfrak{g}[[t]], \mathfrak{g}, \mathbb{V}^{\text {cl }}\right)$. We will prove that all higher differentials vanish. This will imply that, for the PBW filtration,

$$
\operatorname{gr} H^{\bullet}(\mathfrak{g}[[t]], \mathfrak{g}, \mathbb{V})=H^{\bullet}\left(\mathfrak{g}[[t]], \mathfrak{g}, \mathbb{V}^{\mathrm{cl}}\right),
$$

which is described by Theorem 1.2. We will see that the algebra structures agree and will verify the equality gr $\varphi_{\kappa_{0}}=\varphi_{\kappa_{0}}^{\mathrm{cl}}$. This will imply that $H^{\bullet}(\mathfrak{g}[[t]], \mathfrak{g}, \mathbb{V})$ is generated as an algebra in degrees 0 and 1. Theorem 4.1 and Lemma 4.3 will allow us to identify $\varphi_{\kappa_{0}}$ with de Rham's differential to give a linear map

$$
H^{1}(\mathfrak{g}[[t]], \mathfrak{g}, \mathbb{V}) \rightarrow \Omega^{1}\left(\mathrm{Op}_{L_{\mathfrak{g}}}(D)\right)
$$

whose gr is the isomorphism of Theorem 1.2. Symmetrized multiplication ${ }^{3}$ of the generators will give us a linear map in (4.5), whose associated graded is the isomorphism in Theorem 1.2. The theorem will then follow, since we know from Proposition 3.3 and Lemma 3.6 that the algebra $H^{\bullet}(\mathfrak{g}[[t]], \mathfrak{g}, \mathbb{V})$ is skew-commutative, and so (4.5) is an algebra isomorphism.

Now we proceed with the proof. First note that $H^{0}(\mathfrak{g}[[t]], \mathfrak{g}, \mathbb{V})$ is isomorphic to $\operatorname{Fun}\left(\mathrm{Op}_{L_{\mathfrak{g}}}(D)\right)$ according to Theorem 4.1. Since $\operatorname{gr} \operatorname{Fun}\left(\mathrm{Op}_{L_{\mathfrak{g}}}(D)\right)=\operatorname{Fun}\left(C_{\mathfrak{g}^{*}, \omega}\right)$, we find from Theorem 1.2 that the entire zeroth cohomology part of the first term of the spectral sequence survives. (Here and below we say that a class in a particular term of a spectral sequence 'survives' if it is annihilated by all higher differentials of the spectral sequence and does not lie in the image of any of the higher differentials.)

Now let us look at the first cohomology part of the spectral sequence. Recall that

$$
\operatorname{Fun}\left(C_{\mathfrak{g}^{*}, \omega}\right) \simeq \mathbb{C}\left[P_{i, n}\right]_{i=1, \ldots, \ell ; n \geqslant 0} .
$$

Let us choose elements $\widetilde{P}_{i, n}$ in the algebra $\operatorname{Fun}\left(\mathrm{Op}_{L_{\mathfrak{g}}}(D)\right)$ whose symbols are equal to $P_{i, n}$. Then we have

$$
\operatorname{Fun}\left(\mathrm{Op}_{L_{\mathfrak{g}}}(D)\right) \simeq \mathbb{C}\left[\widetilde{P}_{i, n}\right]_{i=1, \ldots, \ell ; n \geqslant 0}
$$

Applying the map $\varphi_{\kappa_{0}}$ to the $\widetilde{P}_{i, n}$, we obtain classes in the first cohomology group $H^{1}(\mathfrak{g}[[t]], \mathfrak{g}, \mathbb{V})$. To show that these classes are non-zero, we compute how the symbol of $\varphi_{\kappa_{0}}$ acts on the associate graded cohomology and find that it coincides with the operator $\varphi_{\kappa_{0}}^{\mathrm{cl}}=\varphi_{\kappa_{0}}^{\mathrm{cl}, 0}$.

Indeed, let us compute how the action of $A_{n}=A \otimes t^{n} \in \mathfrak{g}[[t]]$ on $\mathbb{V}$ changes when we deform the central extension. We find that when we apply this element to a lexicographically ordered monomial $B_{1, m_{1}} \cdots B_{k, m_{k}} v_{\kappa} \in \mathbb{V}_{\kappa}$, the deformation is equal to

$$
h \sum_{i=1}^{k} n \delta_{n,-m_{i}} \kappa_{0}\left(A, B_{i}\right) B_{1, m_{1}} \cdots \widehat{B}_{i, m_{i}} \cdots B_{k, m_{k}} v_{\kappa}
$$

plus the sum of monomials of order less than $k-1$. Thus, the $h$-linear term of the deformation sends the $k$ th term of the PBW filtration on $\mathbb{V}$ to the $(k-1)$ th term, and comparing with formula (1.4), we find that the corresponding operator on the associated graded coincides with the operator $\varphi_{\kappa_{0}}^{\mathrm{cl}}$.

${ }^{3}$ We know from Proposition 3.3 that $H^{\bullet}(\mathfrak{g}[[t]], \mathfrak{g}, \mathbb{V})$ is commutative, but we prefer not to use it here. 


\section{SELF-EXTENSIONS OF VERMa MOdUles AND DifFERENTIAL FORMS ON OPERS}

Therefore we find that the symbol of the cohomology class $\varphi_{\kappa_{0}}\left(\widetilde{P}_{i, n}\right)$ is equal to $\varphi_{\kappa_{0}}^{\mathrm{cl}}\left(P_{i, n}\right)$, which is one of the odd generators of $H^{\bullet}\left(\mathfrak{g}[[t]], \mathfrak{g}, \mathbb{V}^{\text {cl }}\right)$ from $H^{1}\left(\mathfrak{g}[[t]], \mathfrak{g}, \mathbb{V}^{\text {cl }}\right)$. Thus, we obtain that the cocycles representing all odd generators of $H^{\bullet}\left(\mathfrak{g}[[t]], \mathfrak{g}, \mathbb{V}^{\text {cl }}\right)$ may be lifted to cocycles in the Chevalley complex $C^{\bullet}(\mathfrak{g}[[t]], \mathfrak{g}, \mathbb{V})$. Since $H^{\bullet}(\mathfrak{g}[[t]], \mathfrak{g}, \mathbb{V})$ is computed by a spectral sequence whose first term is $H^{\bullet}\left(\mathfrak{g}[[t]], \mathfrak{g}, \mathbb{V}^{\mathrm{cl}}\right)$ we find that these cocycles can become trivial only if their symbols could be obtained from some cohomology classes in $H^{0}\left(\mathfrak{g}[[t]], \mathfrak{g}, \mathbb{V}^{\mathrm{cl}}\right)$ under the action of the higher differentials on our spectral sequence. But we know that all classes in $H^{0}\left(\mathfrak{g}[[t]], \mathfrak{g}, \mathbb{V}^{\mathrm{cl}}\right)$ survive, so under the action of the higher differentials they cannot kill any classes in $H^{1}\left(\mathfrak{g}[[t]], \mathfrak{g}, \mathbb{V}^{\mathrm{cl}}\right)$. Hence all of these classes survive, and we obtain an injective map $C_{\mathfrak{g}^{*}, \omega}^{*} \rightarrow H^{1}(\mathfrak{g}[[t]], \mathfrak{g}, \mathbb{V})$.

The natural action of $H^{0}(\mathfrak{g}[[t]], \mathfrak{g}, \mathbb{V})=\operatorname{End}_{\hat{\mathfrak{g}}_{\kappa_{\mathrm{c}}}} \mathbb{V}$ on $H^{i}(\mathfrak{g}[[t]], \mathfrak{g}, \mathbb{V})$ leads to a map

$$
\operatorname{Fun}\left(\mathrm{Op}_{L_{\mathfrak{g}}}(D)\right) \otimes C_{\mathfrak{g}^{*}, \omega}^{*} \rightarrow H^{1}(\mathfrak{g}[[t]], \mathfrak{g}, \mathbb{V}) .
$$

In the same way as above, we find that it is injective. Considering symbols and comparing with $H^{1}\left(\mathfrak{g}[[t]], \mathfrak{g}, \mathbb{V}^{\mathrm{cl}}\right)$, we find that this map is an isomorphism.

We now generate higher cohomology classes in $H^{i}(\mathfrak{g}[[t]], \mathfrak{g}, \mathbb{V}), i>1$, by taking symmetrized products of the cocycles representing the classes in $H^{1}(\mathfrak{g}[[t]], \mathfrak{g}, \mathbb{V})$ that we have just constructed. We need to prove inductively that the resulting cohomology classes are non-trivial and $\operatorname{span} H^{\bullet}(\mathfrak{g}[[t]]$, $\mathfrak{g}, \mathbb{V})$. In light of the classical result, Theorem 1.2, it suffices to show that the multiplicative structures on $H^{\bullet}(\mathfrak{g}[[t]], \mathfrak{g}, \mathbb{V})$ and $H^{\bullet}\left(\mathfrak{g}[[t]], \mathfrak{g}, \mathbb{V}^{\text {cl }}\right)$ are compatible, in the sense that the latter is the associated graded of the former.

We will give two proofs of this assertion. The first one is to use formulas (3.12) and (3.2) defining the product structure on $H^{\bullet}(\mathfrak{g}[[t]], \mathfrak{g}, \mathbb{V})$ using the structure of vertex algebra. Then we find immediately from the vacuum axiom of vertex algebra that the symbol of the product of two cocycles $A, B \in C^{\bullet}(\mathfrak{g}[[t]], \mathfrak{g}, \mathbb{V})$, i.e. $\operatorname{symb}\left(A_{(-1)} B\right)$, is equal to the product of their symbols, $\operatorname{symb}(A) \operatorname{symb}(B)$.

Now we give another proof that does not use the vertex superalgebra structure on $C^{\bullet}(\mathfrak{g}[[t]], \mathfrak{g}, \mathbb{V})$. Let us introduce a Lie algebra filtration $\left(F_{i}\right)$ on $\mathfrak{g}((t))$ by setting

$$
F_{-1}=0, \quad F_{0}=\mathfrak{g}[[t]], \quad F_{1}=\mathfrak{g}((t)) .
$$

Then the associated graded Lie algebra is

$$
\widetilde{\mathfrak{g}}=\mathfrak{g}[[t]] \oplus \mathfrak{g}((t)) / \mathfrak{g}[[t]],
$$

where the Lie algebra structure on the first summand is the usual one, on the second summand it is commutative, and the commutator between elements of the two summands is given by the natural action of $\mathfrak{g}[[t]]$ on $\mathfrak{g}((t)) / \mathfrak{g}[[t]]$. The induced filtration on $\mathbb{V}$ coincides with the PBW filtration. Hence we have an isomorphism

$$
\operatorname{gr} \mathbb{V} \simeq \operatorname{Ind}_{\mathfrak{g}[[z]]}^{\widetilde{\mathfrak{g}}} \mathbb{C} \simeq \mathbb{V}^{\mathrm{cl}}
$$

Thus, using the Shapiro lemma, we obtain the isomorphism

$$
H^{\bullet}\left(\mathfrak{g}[[t]], \mathfrak{g}, \mathbb{V}^{\mathrm{cl}}\right) \simeq H^{\bullet}\left(\widetilde{\mathfrak{g}}, \mathfrak{g}, \text { End } \mathbb{V}^{\mathrm{cl}}\right),
$$

which is compatible with the multiplicative structure. But the product on the algebra $H^{\bullet}(\widetilde{\mathfrak{g}}, \mathfrak{g}$, End $\left.\mathbb{V}^{\mathrm{cl}}\right)$ is clearly the associated graded of the product on $H^{\bullet}(\mathfrak{g}((t)), \mathfrak{g}$, End $\mathbb{V})$, and hence we obtain the desired assertion.

Finally, it follows from Proposition 3.3 and Lemma 3.6 that the algebra $H^{\bullet}(\mathfrak{g}[[t]], \mathfrak{g}, \mathbb{V})$ is skewcommutative. This completes the proof of Theorem 4.4.

Remark 4.5. The skew-commutativity of $H^{\bullet}(\mathfrak{g}[[t]], \mathfrak{g}, \mathbb{V})$ may be shown without using the vertex superalgebra structure on $C^{\bullet}(\mathfrak{g}[[t]], \mathfrak{g}, \mathbb{V})$. By our construction, $H^{\bullet}(\mathfrak{g}[[t]], \mathfrak{g}, \mathbb{V})$ is generated by classes 


\section{E. Frenkel and C. Teleman}

in $H^{0}$ and $H^{1}$, and we already know that all elements $H^{0}(\mathfrak{g}[[t]], \mathfrak{g}, \mathbb{V})$ come from central elements. Thus, $H^{0}$ is central in the entire cohomology, and it remains to show that the generators in $H^{1}$ anti-commute. These generators have the form $\varphi_{\kappa_{0}}(A)$ where $A \in H^{0}(\mathfrak{g}[[t]], \mathfrak{g}, \mathbb{V})$. Their anticommutativity follows from Lemma 4.3. Indeed, we have (denoting $\varphi_{\kappa_{0}}$ by $\delta_{1}$ as in the proof of Lemma 4.3)

$$
\left[\delta_{1}(A), \delta_{1}(B)\right]_{+}=\delta_{1}\left(\left[A, \delta_{1}(B)\right]\right)-A \delta_{1}^{2}(B)+\delta_{1}^{2}(B) A,
$$

using the Leibniz rule. The first term on the right-hand side vanishes because $A$ is central in $H^{\bullet}(\mathfrak{g}[[t]], \mathfrak{g}, \mathbb{V})$, and the remaining two terms vanish because $\delta_{1}^{2}=0$ on the cohomology. Hence $\left[\delta_{1}(A), \delta_{1}(B)\right]_{+}=0$.

Proof of Proposition 4.2. Consider the spectral sequence induced by the PBW filtration on $\mathbb{V}_{\kappa}$ and converging to $H^{\bullet}\left(\mathfrak{g}[[t]], \mathfrak{g}, \mathbb{V}_{\kappa}\right)$. It has the same first term $H^{\bullet}\left(\mathfrak{g}[[t]], \mathfrak{g}, \mathbb{V}^{\text {cl }}\right)$ as the one for $\mathbb{V}$, considered in the proof of Theorem 4.4. The computation of $\varphi_{\kappa_{0}}$ in the above proof shows that the first differential of this spectral sequence for $\mathbb{V}_{\kappa}$ differs from the one for $\mathbb{V}$ by the map $h \varphi_{\kappa_{0}}^{\mathrm{cl}}$, where $\kappa=\kappa_{0}+h \kappa_{0}$ (see (4.6)). But the differential for $\mathbb{V}$ was equal to zero, and we know from Theorem 1.2 that $\varphi_{\kappa_{0}}^{\mathrm{cl}}$ is the de Rham differential on

$$
H^{\bullet}\left(\mathfrak{g}[[t]], \mathfrak{g}, \mathbb{V}^{\mathrm{cl}}\right) \simeq \Omega^{\bullet}\left(C_{\mathfrak{g}^{*}, \omega}\right) .
$$

Hence its cohomology is $\mathbb{C}$, in degree 0 .

Next, we consider the absolute cohomology.

Proposition 4.6. The cohomology $H^{\bullet}(\mathfrak{g}[[t]], \mathbb{V})$ is canonically isomorphic to the tensor product of $H^{\bullet}(\mathfrak{g}[[t]], \mathfrak{g}, \mathbb{V})$ described in Theorem 4.4 and $H^{\bullet}(\mathfrak{g}, \mathbb{C})$ which is the exterior algebra with generators in degrees $2 d_{i}+1, i=1, \ldots, \ell$.

Proof. The argument is standard, applying to any semi-direct product Lie algebra $\mathfrak{g} \ltimes \mathfrak{L}(\mathfrak{L}=$ $\mathfrak{g} \otimes t \mathbb{C}[[t]]$ here), provided that $\mathfrak{g}$ is reductive, $\mathfrak{L}$ is a direct product, and $\mathbb{V}$ is a direct sum of finite-dimensional irreducible $\mathfrak{g}$-modules.

Consider the Serre-Hochshild spectral sequence corresponding to the Lie subalgebra $\mathfrak{g} \subset \mathfrak{g}[[t]]$ (see e.g. [Fuc88]). In the first term we have

$$
E_{1}^{p, q}=H^{q}\left(\mathfrak{g}, \mathbb{V} \otimes \bigwedge^{p}(\mathfrak{g}[[t]] / \mathfrak{g})^{*}\right)=H^{q}(\mathfrak{g}, \mathbb{C}) \otimes C^{p}(\mathfrak{g}[[t]], \mathfrak{g}, \mathbb{V}),
$$

where

$$
C^{p}(\mathfrak{g}[[t]], \mathfrak{g}, \mathbb{V})=\left(\mathbb{V} \otimes \bigwedge^{p}(\mathfrak{g}[[t]] / \mathfrak{g})^{*}\right)^{\mathfrak{g}}
$$

is the $p$ th group of the relative Chevalley complex computing $H^{\bullet}(\mathfrak{g}[[t]], \mathfrak{g}, \mathbb{V})$. Therefore in the second term we have

$$
E_{2}^{p, q}=H^{q}(\mathfrak{g}, \mathbb{C}) \otimes H^{p}(\mathfrak{g}[[t]], \mathfrak{g}, \mathbb{V}) .
$$

We can therefore represent classes in $E_{2}^{p, q}$ as tensor products $\omega_{1} \otimes \omega_{2}$ of a cocycle $\omega_{1}$ in $C^{q}(\mathfrak{g}, \mathbb{C})$ representing a class in $H^{q}(\mathfrak{g}, \mathbb{C})$ and a cocycle $\omega_{2}$ in $\left(\mathbb{V} \otimes \bigwedge^{p}(\mathfrak{g} \otimes t \mathbb{C}[[t]])^{*}\right)^{\mathfrak{g}}$ representing a class in $H^{p}(\mathfrak{g}[[t]], \mathfrak{g}, \mathbb{V})$. Applying the differential to this class, we find that it is identically equal to zero because $\omega_{2}$ is $\mathfrak{g}$-invariant. Therefore all the classes in $E_{2}$ survive. Moreover, all of the generators of the two factors in the decomposition (4.7) lift canonically to the cohomology $H^{\bullet}(\mathfrak{g}[[t]], \mathbb{V})$, and so we obtain the desired statement. 


\section{SELF-EXTENSIONS OF VERMa MODUles AND DIFFERENTIAL FORMS ON OPERS}

\section{Cohomology of the classical Verma module}

Let $\mathfrak{b}$ be a Borel subalgebra of $\mathfrak{g}$. Denote by $\widetilde{\mathfrak{b}}$ the Lie subalgebra of $\mathfrak{g}[[t]]$ which is the preimage of $\mathfrak{b}$ under the evaluation homomorphism $\mathfrak{g}[[t]] \rightarrow \mathfrak{g}$. Thus, we can write

$$
\widetilde{\mathfrak{b}}=\mathfrak{b} \otimes 1 \oplus \mathfrak{g} \otimes t \mathbb{C}[[t]] .
$$

The Lie group of $\widetilde{\mathfrak{b}}$ is the preimage of $B$, the Borel subgroup of $G$ corresponding to $\mathfrak{b}$ under the evaluation homomorphism $G[[t]] \rightarrow G$. We call it the Iwahori subgroup and denote it by $\widetilde{B}$.

Decompose $\mathfrak{b}=\mathfrak{h} \oplus \mathfrak{n}$ into a direct sum of a Cartan subalgebra and a nilpotent subalgebra. Then we have an analogous decomposition of $\widetilde{\mathfrak{b}}: \widetilde{\mathfrak{b}}=\mathfrak{h} \oplus \widetilde{\mathfrak{n}}$, where

$$
\widetilde{\mathfrak{n}}=\mathfrak{n} \otimes 1 \oplus \mathfrak{g} \otimes t \mathbb{C}[[t]] .
$$

Given $\lambda \in \mathfrak{h}^{*}$, let $\mathbb{C}_{\lambda}$ be the one-dimensional representation of the Lie subalgebra $\widetilde{\mathfrak{b}} \oplus \mathbb{C} K$ of $\hat{\mathfrak{g}}_{\kappa}$, on which $\widetilde{\mathfrak{n}}$ acts by $0, \mathfrak{h}$ acts according to $\lambda$ and $K$ acts as the identity. Then the Verma module $\mathbb{M}_{\lambda, \kappa}$ of highest weight $\lambda$ at level $\kappa$ is by definition the induced module

$$
\mathbb{M}_{\lambda, \kappa}=\operatorname{Ind}_{\widetilde{\mathfrak{b}} \oplus \mathbb{C} K}^{\hat{\mathfrak{a}}_{\kappa}} \mathbb{C}_{\lambda} .
$$

Let $H$ be the Cartan subgroup of $G$ whose Lie algebra is $\mathfrak{h}$. We have the categories $H C\left(\hat{\mathfrak{g}}_{\kappa}, H\right)$ and $H C\left(\hat{\mathfrak{g}}_{\kappa}, \widetilde{B}\right)$ of continuous Harish-Chandra modules corresponding to the pairs $\left(\hat{\mathfrak{g}}_{\kappa}, H\right)$ and $\left(\hat{\mathfrak{g}}_{\kappa}, \widetilde{B}\right)$, respectively.

We wish to compute the algebras of self-extensions

$$
\operatorname{Ext}_{H C\left(\hat{\mathfrak{g}}_{\kappa}, H\right)}^{\bullet}\left(\mathbb{M}_{\lambda, \kappa}, \mathbb{M}_{\lambda, \kappa}\right) \quad \text { and } \quad \operatorname{Ext}_{H C\left(\hat{\mathfrak{g}}_{\kappa}, \widetilde{B}\right)}^{\bullet}\left(\mathbb{M}_{\lambda, \kappa}, \mathbb{M}_{\lambda, \kappa}\right) .
$$

Consider the relative cohomology

$$
H^{\bullet}\left(\mathfrak{g}((t)), \mathfrak{h}, \text { End } \mathbb{M}_{\lambda, \kappa}\right)
$$

with its natural algebra structure. In the same way as in $\S 2$, we obtain the following result.

Proposition 5.1. There are natural isomorphisms of algebras

$$
\operatorname{Ext}_{H C\left(\hat{\mathfrak{g}}_{\kappa}, H\right)}^{\bullet}\left(\mathbb{M}_{\lambda, \kappa}, \mathbb{M}_{\lambda, \kappa}\right) \simeq \operatorname{Ext}_{H C\left(\hat{\mathfrak{g}}_{\kappa}, \widetilde{B}\right)}^{\bullet}\left(\mathbb{M}_{\lambda, \kappa}, \mathbb{M}_{\lambda, \kappa}\right) \simeq H^{\bullet}\left(\mathfrak{g}((t)), \mathfrak{h}, \text { End } \mathbb{M}_{\lambda, \kappa}\right)
$$

Next, consider the relative cohomology

$$
H^{\bullet}\left(\widetilde{\mathfrak{b}}, \mathfrak{h}, \mathbb{M}_{\lambda, \kappa} \otimes \mathbb{C}_{-\lambda}\right)=H^{\bullet}\left(\widetilde{\mathfrak{n}}, M_{\lambda, \kappa}\right)_{\lambda},
$$

where on the right-hand side we consider the $\lambda$-component of the cohomology $H^{\bullet}\left(\widetilde{\mathfrak{n}}, \mathbb{M}_{\lambda, \kappa_{\mathrm{c}}}\right)$ with respect to the natural action of the Cartan subalgebra $\mathfrak{h}$. Using the Shapiro lemma as in Lemma 3.1, we obtain an isomorphism

$$
H^{\bullet}\left(\widetilde{\mathfrak{b}}, \mathfrak{h}, \mathbb{M}_{\lambda, \kappa} \otimes \mathbb{C}_{-\lambda}\right) \simeq H^{\bullet}\left(\mathfrak{g}((t)), \mathfrak{h}, \text { End } \mathbb{M}_{\lambda, \kappa}\right) .
$$

This isomorphism gives an algebra structure to $H^{\bullet}\left(\widetilde{\mathfrak{b}}, \mathfrak{h}, \mathbb{M}_{\lambda, \kappa} \otimes \mathbb{C}_{-\lambda}\right)$.

Thus, to compute the algebras of self-extensions of $\mathbb{M}_{\lambda, \kappa}$ introduced above, we need to compute the relative Lie algebra cohomologies $H^{\bullet}\left(\widetilde{\mathfrak{b}}, \mathfrak{h}, \mathbb{M}_{\lambda, \kappa} \otimes \mathbb{C}_{-\lambda}\right)$. The computation will proceed in the same way as in the case of the vacuum module. Namely, we will use a spectral sequence whose first term is the cohomology of the graded version of $\mathbb{M}_{\lambda, \kappa}$. In the rest of this section we describe the latter.

The $\widetilde{\mathfrak{b}}$-module $\mathbb{M}_{\lambda, \kappa} \otimes \mathbb{C}_{-\lambda}$ carries a PBW filtration and the associate graded is isomorphic, as a $\widetilde{\mathfrak{b}}$-module, to

$$
\mathbb{M}^{\mathrm{cl}}=\operatorname{Sym}(\mathfrak{g}((t)) / \widetilde{\mathfrak{b}}) .
$$




\section{E. Frenkel and C. Teleman}

We consider the relative cohomology $H^{\bullet}\left(\widetilde{\mathfrak{b}}, \mathfrak{h}, \mathbb{M}^{\text {cl }}\right)$. This cohomology is computed by the standard Chevalley complex of Lie algebra cohomology

$$
C^{\bullet}\left(\widetilde{\mathfrak{b}}, \mathfrak{h}, \mathbb{M}^{\mathrm{cl}}\right)=\left(\mathbb{M}^{\mathrm{cl}} \otimes \bigwedge^{\bullet} \widetilde{\mathfrak{n}}^{*}\right)^{\mathfrak{h}}=\left(\operatorname{Sym}(\mathfrak{g}((t)) / \widetilde{\mathfrak{b}}) \otimes \bigwedge^{\bullet} \widetilde{\mathfrak{n}}^{*}\right)^{\mathfrak{h}}
$$

The algebra structure on $\mathbb{M}^{\mathrm{cl}}$ gives rise to a graded algebra structure on the cohomology of this complex. We compute this cohomology using Theorem 1.2.

Recall the space $C_{\mathfrak{g}^{*}}$ from $\S 1$ and define $C_{\mathfrak{g}^{*}, \omega}^{\mathrm{RS}}$ as

$$
C_{\mathfrak{g}^{*}, \omega}^{\mathrm{RS}}=\Gamma\left(D,\left(\omega(0) \underset{\mathbb{C}^{\times}}{\times} C_{\mathfrak{g}^{*}}\right)(-0)\right) \simeq t\left(t^{-1} \mathbb{C}[[t]] d t \underset{\mathbb{C}^{\times}}{\times} C_{\mathfrak{g}^{*}}\right),
$$

where 0 is the closed point of the disc $D=\operatorname{Spec} \mathbb{C}[[t]]$ and $(-0)$ indicates sections sending the closed point of $D$ to the origin of the cone. Using the generators $P_{i}, i=1, \ldots, \ell$, of $\left(\text { Fun } \mathfrak{g}^{*}\right)^{G}$ introduced in $\S 1$, we obtain an identification

$$
\operatorname{Fun}\left(C_{\mathfrak{g}^{*}, \omega}^{\mathrm{RS}}\right)=\mathbb{C}\left[P_{i, n}\right]_{i=1, \ldots, \ell ; n \geqslant-d_{i}},
$$

where the $P_{i, n}$ are the functions on $\mathfrak{g}^{*} \otimes t^{-1} \mathbb{C}[[t]] d t$ defined by (1.1). Thus, we obtain a coordinateindependent isomorphism

$$
C_{\mathfrak{g}^{*}, \omega}^{\mathrm{RS}} \simeq \bigoplus_{i=1}^{\ell} \Gamma\left(D, \omega^{\otimes\left(d_{i}+1\right)}\left(d_{i} \cdot 0\right)\right)=t^{-d_{i}} \mathbb{C}[[t]](d t)^{\otimes\left(d_{i}+1\right)} .
$$

Consider the restrictions of the functions $P_{i, n}$ to

$$
(\mathfrak{g}((t)) / \widetilde{\mathfrak{b}})^{*} \subset(\mathfrak{g}((t)) / \mathfrak{g} \otimes t \mathbb{C}[[t]])^{*} \simeq \mathfrak{g}^{*} \otimes t^{-1} \mathbb{C}[[t]] d t .
$$

Since by construction these functions are $\widetilde{b}$-invariant, we obtain a map

$$
\operatorname{Fun}\left(C_{\mathfrak{g}^{*}, \omega}^{\mathrm{RS}}\right) \rightarrow H^{0}\left(\widetilde{\mathfrak{b}}, \mathfrak{h}, \mathbb{M}^{\mathrm{cl}}\right) .
$$

Next, we use the map $\varphi_{\kappa_{0}}^{\mathrm{cl}}$ from $\S 1$ to construct a map

$$
\left(C_{\mathfrak{g}^{*}, \omega}^{\mathrm{RS}}\right)^{*} \rightarrow H^{1}\left(\widetilde{\mathfrak{b}}, \mathfrak{h}, \mathbb{M}^{\mathrm{cl}}\right) .
$$

Proposition 5.2. There is a canonical isomorphism of graded algebras

$$
H^{\bullet}\left(\widetilde{\mathfrak{b}}, \mathfrak{h}, \mathbb{M}^{\mathrm{cl}}\right) \simeq \Omega^{\bullet}\left(C_{\mathfrak{g}^{*}, \omega}^{\mathrm{RS}}\right)=\operatorname{Fun}\left(C_{\mathfrak{g}^{*}, \omega}^{\mathrm{RS}}\right) \otimes \bigwedge^{\bullet}\left(C_{\mathfrak{g}, \omega}^{\mathrm{RS}}\right)^{*}
$$

The right-hand side is a free skew-commutative algebra with the even generators $P_{i, n} \in H^{0}\left(\widetilde{\mathfrak{b}}, \mathfrak{h}, \mathbb{M}^{\mathrm{cl}}\right)$ defined by (1.1) and the odd generators $\varphi_{\kappa_{0}}^{\mathrm{cl}}\left(P_{i, n}\right) \in H^{1}\left(\widetilde{\mathfrak{b}}, \mathfrak{h}, \mathbb{M}^{\mathrm{cl}}\right)$ defined by $(1.2)$, where $i=1, \ldots, \ell$ and $n \geqslant-d_{i}$.

Proof. We use the same argument as in the proof of Theorem 1.13 of [FGT04], where a closely related cohomology, $H^{\bullet}(\widetilde{\mathfrak{b}}, \mathfrak{h}, \operatorname{Sym}(\mathfrak{g}((t)) / \widetilde{\mathfrak{n}}))$, was computed.

Using the van Est spectral sequence, we obtain that

$$
H^{\bullet}\left(\widetilde{\mathfrak{b}}, \mathfrak{h}, \mathbb{M}^{\mathrm{cl}}\right) \simeq H_{\widetilde{B}}^{\bullet}\left(\mathbb{M}^{\mathrm{cl}}\right), \quad H^{\bullet}\left(\mathfrak{g}[[t]], \mathfrak{g}, \mathbb{V}^{\mathrm{cl}}\right) \simeq H_{G[[t]]}^{\bullet}\left(\mathbb{V}^{\mathrm{cl}}\right)
$$

By the Shapiro lemma, we have a spectral sequence converging to $H_{\widetilde{B}}^{\bullet}\left(\mathbb{M}^{\mathrm{cl}}\right)$ whose second term consists of the cohomologies

$$
H_{G[[t]]}^{p}\left(R^{q} \operatorname{Ind}_{\widetilde{B}}^{G[[t]]} \mathbb{M}^{\mathrm{cl}}\right)
$$

Using the residue pairing and a non-degenerate inner product $\kappa_{0}$ on $\mathfrak{g}$ we have an isomorphism

$$
\mathfrak{g}((t)) / \widetilde{\mathfrak{b}} \simeq \widetilde{\mathfrak{n}} \frac{d t}{t} \simeq \widetilde{\mathfrak{n}}
$$


as a $\widetilde{\mathfrak{b}}$-module, and hence

$$
\mathbb{M}^{\mathrm{cl}}=\operatorname{Sym}(\mathfrak{g}((t)) / \widetilde{\mathfrak{b}}) \simeq \operatorname{Fun}(\widetilde{\mathfrak{n}})
$$

Therefore we have

$$
R^{q} \operatorname{Ind}_{\widetilde{B}}^{G[[t]]} \mathbb{M}^{\mathrm{cl}}=H^{q}(G[[t]] \underset{\widetilde{B}}{\times} \widetilde{\mathfrak{n}}, \mathcal{O}),
$$

where $\mathcal{O}$ is the structure sheaf. But the splitting $G[[t]]=G \times G^{(1)}$, where $G^{(1)}$ is the first congruence subgroup, gives rise to the following isomorphism of $G$-equivariant vector bundles over $G[[t]] / \widetilde{B} \simeq$ $G / B$ :

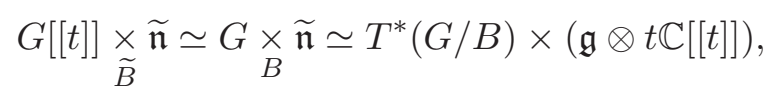

where the second isomorphism is due to the fact that

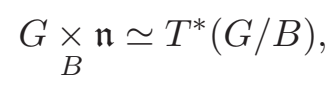

and the second summand in the direct sum decomposition (5.1) is a $G$-module giving rise to a trivial vector bundle on $G / B$.

It follows from the results of [Hes76] that

$$
H^{q}\left(T^{*}(G / B), \mathcal{O}\right)=0, \quad q>0,
$$

and

$$
H^{0}\left(T^{*}(G / B), \mathcal{O}\right)=\operatorname{Fun}(\mathcal{N}),
$$

where $\mathcal{N} \subset \mathfrak{g}$ is the nilpotent cone and the functions on $\mathcal{N}$ are pulled back to $T^{*}(G / B)$ via the moment map $T^{*}(G / B) \rightarrow \mathfrak{g}^{*} \stackrel{\kappa_{0}}{\longrightarrow} \mathfrak{g}$ (its image belongs to $\mathcal{N}$ ). This implies that $R^{q} \operatorname{Ind}_{\widetilde{B}}^{G[t]]} \mathbb{M}^{\text {cl }}=0$ for $q>0$. Consider the morphism

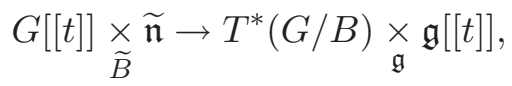

$$
\begin{aligned}
& (g, x) \mapsto((\bar{g}, \overline{g(x)}), g(x)),
\end{aligned}
$$

where $\bar{g}$ is the projection of $g \in G[[t]]$ onto $G[[t]] / \widetilde{B} \simeq G / B$ and $\overline{g(x)}=g(x) \bmod \mathfrak{g} \otimes t \mathbb{C}[[t]]$, so that $\overline{g(x)} \in T_{\bar{g}}^{*}(G / B)$. It is clear that this is an isomorphism, and so we find that

$$
\operatorname{Ind}_{\widetilde{B}}^{G[[t]]} \mathbb{M}^{\mathrm{cl}} \simeq \operatorname{Fun}(G[[t]] \underset{\widetilde{B}}{\times \widetilde{\mathfrak{n}}}) \simeq \operatorname{Fun}(\mathcal{N} \times \underset{\mathfrak{g}}{\times \mathfrak{g}} \mathfrak{g}[[t]])
$$

Therefore we obtain that

$$
H_{\widetilde{B}}^{p}\left(\mathbb{M}^{\mathrm{cl}}\right)=H_{G[[t]]}^{p}(\operatorname{Fun}(\mathcal{N} \times \underset{\mathfrak{g}}{\mathfrak{g}}[[t]])) .
$$

Recall that $\mathcal{N}$ is a complete intersection whose ideal in Fun $\mathfrak{g}$ is the augmentation ideal of the ring of invariant functions $(\text { Fun } \mathfrak{g})^{G}$. Thus, the ring of functions on the fiber product appearing on the right-hand side may be resolved by the Koszul complex

$$
\text { Fun } \mathfrak{g} \otimes \bigwedge^{\bullet}\left(P_{i}\right)_{i=1, \ldots, \ell}
$$

Hence we find that $H^{\bullet}\left(\widetilde{\mathfrak{b}}, \mathfrak{h}, \mathbb{M}^{\mathrm{cl}}\right)$ is equal to the cohomology of the double complex

$$
H^{\bullet}(\mathfrak{g}[[t]], \mathfrak{g}, \operatorname{Fun}(\mathfrak{g}[[t]])) \otimes \bigwedge^{\bullet}\left(P_{i}\right)_{i=1, \ldots, \ell} .
$$

But we already know the first factor from Theorem 1.2. It is clear from the description of the generators of this cohomology that the even generators $P_{i, 0}$ get eliminated by the exterior algebra $\bigwedge^{\bullet}\left(P_{i}\right)_{i=1, \ldots, \ell}$. Hence we obtain that $H^{\bullet}\left(\widetilde{\mathfrak{b}}, \mathfrak{h}, \mathbb{M}^{\mathrm{cl}}\right)$ is a free skew-commutative algebra generated by all remaining generators as described in Theorem 1.2. This gives us the statement of the proposition, 


\section{E. Frenkel and C. Teleman}

with the shifting of the labeling of the generators due to the fact that we have used the identification $\widetilde{\mathfrak{n}} d t / t \simeq \widetilde{\mathfrak{n}}$ in the course of the proof.

\section{Self-extensions of the Verma modules}

We can now compute the algebras of self-extensions of the Verma modules $\mathbb{M}_{\lambda, \kappa}$ in the categories of Harish-Chandra modules introduced above. First, we describe some deformations of the space $C_{\mathfrak{g}^{*}, \omega}^{\mathrm{RS}}$.

Following [BD05, $§ 3.8 .8]$, define a ${ }^{L} \mathfrak{g}$-oper with regular singularity as the equivalence class operators of the form

$$
\partial_{t}+\frac{1}{t}\left(p_{-1}+\mathbf{v}(t)\right), \quad \mathbf{v}(t) \in{ }^{L} \mathfrak{b}[[t]]
$$

with respect to the gauge action of ${ }^{L} N[[t]]$. Define the residue of this oper as $p_{-1}+\mathbf{v}(0)$. Clearly, under gauge transformations by an element $x(t)$ of ${ }^{L} N[[t]]$ the residue gets conjugated by $x(0) \in{ }^{L} N$. Therefore its projection onto ${ }^{L} \mathfrak{g} /{ }^{L} G={ }^{L} \mathfrak{h} / W=\operatorname{Spec}\left(\operatorname{Fun}{ }^{L} \mathfrak{h}\right){ }^{W}$ is well defined. Hence the residue is a point in ${ }^{L} \mathfrak{h} / W=\mathfrak{h}^{*} / W$. Denote the space of ${ }^{L} \mathfrak{g}$-opers with regular singularity by $\mathrm{Op}_{L}^{\mathrm{RS}}(D)$ and for $\lambda \in \mathfrak{h}^{*}$ denote by $\mathrm{Op}_{L_{\mathfrak{g}}}^{\mathrm{RS}}(D)_{\lambda}$ its subspace of opers with residue equal to the projection of $\lambda$ onto $\mathfrak{h}^{*} / W$.

The natural map $\mathrm{Op}_{L_{\mathfrak{g}}}^{\mathrm{RS}}(D)_{\lambda} \rightarrow \mathrm{Op}_{L_{\mathfrak{g}}}\left(D^{\times}\right)$is an embedding and the canonical form of an oper in $\mathrm{Op}_{L_{\mathfrak{g}}}^{\mathrm{RS}}(D)_{\lambda}$ is given by the formula

$$
\partial_{t}+p_{-1}+\sum_{i=1}^{\ell} t^{-d_{i}-1} c_{j}(t) p_{j}, \quad c_{i}(t) \in \mathbb{C}[[t]] .
$$

Moreover, the values $c_{i}(0)$ are uniquely determined by the requirement that $\lambda \in{ }^{L} \mathfrak{h}$ is ${ }^{L} G$-conjugate to

$$
p_{-1}+\left(c_{1}(0)+\frac{1}{4}\right) p_{1}+\sum_{i=2}^{\ell} c_{j}(0) p_{j}
$$

(see $\left[\mathrm{BD} 05\right.$, Proposition 3.8.9]). It follows that the algebra $\operatorname{Fun}\left(\mathrm{Op}_{L_{\mathfrak{g}}}^{\mathrm{RS}}(D)\right)$ has a natural filtration, and the associated graded algebra is isomorphic to $\operatorname{Fun}\left(C_{\mathfrak{g}^{*}, \omega}^{\mathrm{RS}}\right)$.

According to the results of Frenkel (see [Fre04, Theorem 12.4, Lemma 9.4 and Proposition 12.8]), we have the following analogue of Theorem 4.1 for Verma modules.

Theorem 6.1. For any $\lambda \in \mathfrak{h}^{*}$ there is a canonical isomorphism of algebras

$$
\operatorname{End}_{\hat{\mathfrak{g}}_{\kappa_{\mathrm{c}}}} \mathbb{M}_{\lambda, \kappa_{\mathrm{c}}}=H^{0}\left(\widetilde{\mathfrak{b}}, \mathfrak{h}, \mathbb{M}_{\lambda, \kappa_{\mathrm{c}}} \otimes \mathbb{C}_{-\lambda}\right) \simeq \operatorname{Fun}\left(\mathrm{Op}_{L_{\mathfrak{g}}}^{\mathrm{RS}}(D)_{-\lambda-\rho}\right)
$$

The main step in the proof is proving that the associated graded of $\left(\mathbb{M}_{\lambda, \kappa_{\mathrm{c}}} \otimes \mathbb{C}_{-\lambda}\right)^{\widetilde{\mathfrak{b}}}$ with respect to the PBW filtration is equal to $\left(\mathbb{M}^{\mathrm{cl}}\right)^{\widetilde{\mathfrak{b}}}$. We prove this by constructing $\widetilde{\mathfrak{b}}$-invariant vectors in $\mathbb{M}_{\lambda, \kappa_{\mathrm{c}}} \otimes \mathbb{C}_{-\lambda}$ using central elements of the completed universal enveloping algebra of $\hat{\mathfrak{g}}_{\kappa_{\mathrm{c}}}$. Since the center is 'large', namely, it is isomorphic to the algebra of functions on $\mathrm{Op}_{L_{\mathfrak{g}}}\left(D^{\times}\right)$, we construct sufficiently many invariant vectors this way. But we know from Proposition 5.2 that

$$
\left(\mathbb{M}^{\mathrm{cl}}\right)^{\widetilde{\mathfrak{b}}} \simeq \operatorname{Fun}\left(C_{\mathfrak{g}^{*}, \omega}^{\mathrm{RS}}\right) .
$$

Therefore Spec $\operatorname{End}_{\hat{\mathfrak{g}}_{\kappa_{\mathrm{c}}}} \mathbb{M}_{\lambda, \kappa_{\mathrm{c}}}$ is an affine subspace of $\mathrm{Op}_{L_{\mathfrak{g}}}\left(D^{\times}\right)$on which $C_{\mathfrak{g}^{*}, \omega}^{\mathrm{RS}}$ acts simply transitively. The fact that this subspace is equal to $\operatorname{Op}_{L_{\mathfrak{g}}}^{\mathrm{RS}}(D)_{-\lambda-\rho}$ follows from our knowledge of how the degree zero part of the center acts on the highest weight vector of $\mathbb{M}_{\lambda, \kappa_{\mathrm{c}}}$ (see [Fre04, Proposition 12.8]). 


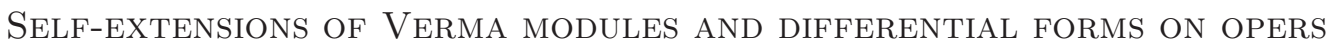

As in the case of the vacuum module, we now generalize Theorem 6.1 to a complete description of the algebra $H^{\bullet}\left(\widetilde{\mathfrak{b}}, \mathfrak{h}, \mathbb{M}_{\lambda, \kappa_{\mathrm{c}}} \otimes \mathbb{C}_{-\lambda}\right)$. It turns out to be isomorphic to the algebra of differential forms on $\mathrm{Op}_{L}^{\mathrm{RS}}(D)_{-\lambda-\rho}$.

TheOREM 6.2. For each non-zero invariant inner product $\kappa_{0}$ on $\mathfrak{g}$ there is an isomorphism of graded algebras

$$
\begin{aligned}
\operatorname{Ext}_{H C\left(\hat{\mathfrak{g}}_{\kappa_{\mathrm{c}}}, \widetilde{B}\right)}^{\bullet}\left(\mathbb{M}_{\lambda, \kappa_{\mathrm{c}}}, \mathbb{M}_{\lambda, \kappa_{\mathrm{c}}}\right) & =H^{\bullet}\left(\widetilde{\mathfrak{b}}, \mathfrak{h}, \mathbb{M}_{\lambda, \kappa_{\mathrm{c}}} \otimes \mathbb{C}_{-\lambda}\right) \\
& \simeq \Omega^{\bullet}\left(\mathrm{Op}_{L_{\mathfrak{g}}}^{\mathrm{RS}}(D)_{-\lambda-\rho}\right) \\
& =\operatorname{Fun}\left(\mathrm{Op}_{L_{\mathfrak{g}}}^{\mathrm{RS}}(D)_{-\lambda-\rho}\right) \otimes \bigwedge^{\bullet}\left(C_{\mathfrak{g}^{*}, \omega}^{\mathrm{RS}}\right)^{*},
\end{aligned}
$$

where $\Omega^{\bullet}\left(\mathrm{Op}_{L_{\mathfrak{g}}}^{\mathrm{RS}}(D)_{-\lambda-\rho}\right)$ is the algebra of differential forms on $\mathrm{Op}_{L_{\mathfrak{g}}}^{\mathrm{RS}}(D)_{-\lambda-\rho}$. If we rescale $\kappa_{0}$ by $\lambda$, then this isomorphism gets rescaled by $\lambda^{-i}$ on the ith cohomology.

Proof. We apply verbatim the proof of Theorem 4.4. We compute the cohomology $H^{\bullet}\left(\widetilde{\mathfrak{b}}, \mathfrak{h}, \mathbb{M}_{\lambda, \kappa_{\mathrm{c}}} \otimes\right.$ $\mathbb{C}_{-\lambda}$ ) using the spectral sequence associated to the PBW filtration on $\mathbb{M}_{\lambda, \kappa_{\mathrm{c}}}$. Its first term is the cohomology $H^{\bullet}\left(\widetilde{\mathfrak{b}}, \mathfrak{h}, \mathbb{M}^{\mathrm{cl}}\right)$ computed in Proposition 5.2. We find from Theorem 6.1 that the zeroth cohomology part of the first term survives. Next, we construct cohomology classes in $H^{1}\left(\widetilde{\mathfrak{b}}, \mathfrak{h}, \mathbb{M}_{\lambda, \kappa_{\mathrm{c}}} \otimes\right.$ $\mathbb{C}_{-\lambda}$ ) in the same way as in Theorem 4.4 , by deforming the module $\mathbb{M}_{\lambda, \kappa_{\mathrm{c}}}$ away from the critical level.

In the same way as in the case of the vacuum module we construct maps

$$
\widetilde{\varphi}_{\kappa_{0}}^{i}: H^{i}\left(\widetilde{\mathfrak{b}}, \mathfrak{h}, \mathbb{M}_{\lambda, \kappa_{\mathrm{c}}} \otimes \mathbb{C}_{-\lambda}\right) \rightarrow H^{i+1}\left(\widetilde{\mathfrak{b}}, \mathfrak{h}, \mathbb{M}_{\lambda, \kappa_{\mathrm{c}}} \otimes \mathbb{C}_{-\lambda}\right) .
$$

Considering the symbols of the classes in $H^{1}\left(\widetilde{\mathfrak{b}}, \mathfrak{h}, \mathbb{M}_{\lambda, \kappa_{\mathrm{c}}} \otimes \mathbb{C}_{-\lambda}\right)$ obtained by applying $\widetilde{\varphi}_{\kappa_{0}}^{0}$ to the generators of $H^{0}\left(\widetilde{\mathfrak{b}}, \mathfrak{h}, \mathbb{M}_{\lambda, \kappa_{\mathrm{c}}} \otimes \mathbb{C}_{-\lambda}\right)$, we find that these are the generators of $H^{1}\left(\widetilde{\mathfrak{b}}, \mathfrak{h}, \mathbb{M}^{\mathrm{cl}}\right)$. Hence these classes survive.

We then use the product structure to produce cohomology classes of higher degrees. We check in the same way as in the proof of Theorem 4.4 (the second argument) that the product on $H^{\bullet}\left(\widetilde{\mathfrak{b}}, \mathfrak{h}, \mathbb{M}_{\lambda, \kappa_{\mathrm{c}}} \otimes \mathbb{C}_{-\lambda}\right)$ is compatible with that on $H^{\bullet}\left(\widetilde{\mathfrak{b}}, \mathfrak{h}, \mathbb{M}^{\mathrm{cl}}\right)$. Therefore we find that all of these classes survive. Finally, we show that the generators of $H^{1}\left(\widetilde{\mathfrak{b}}, \mathfrak{h}, \mathbb{M}_{\lambda, \kappa_{\mathrm{c}}} \otimes \mathbb{C}_{-\lambda}\right)$ anti-commute using the same computation as in Theorem 4.4. This completes the proof.

Finally, we describe the algebras of self-extensions of the Verma modules $\mathbb{M}_{\lambda, \kappa}$ of an arbitrary level $\kappa \neq \kappa_{\mathrm{c}}$.

Proposition 6.3. For $\kappa \neq \kappa_{0}$ and any $\lambda \in \mathfrak{h}^{*}$ we have

$$
\operatorname{Hom}_{\mathfrak{g}_{\kappa}}\left(\mathbb{M}_{\lambda, \kappa}, \mathbb{M}_{\lambda, \kappa}\right)=H^{0}\left(\widetilde{\mathfrak{b}}, \mathfrak{h}, \mathbb{M}_{\lambda, \kappa} \otimes \mathbb{C}_{-\lambda}\right)=\mathbb{C}
$$

and

for all $i>0$.

$$
\operatorname{Ext}_{H C\left(\hat{\mathfrak{g}}_{\kappa}, \widetilde{B}\right)}^{i}\left(\mathbb{M}_{\lambda, \kappa}, \mathbb{M}_{\lambda, \kappa}\right)=H^{i}\left(\widetilde{\mathfrak{b}}, \mathfrak{h}, \mathbb{M}_{\lambda, \kappa} \otimes \mathbb{C}_{-\lambda}\right)=0
$$

Proof. The proof is the verbatim repetition of the proof of Proposition 4.2. First of all, for generic $\kappa$ and $\lambda$ the module $\mathbb{M}_{\lambda, \kappa}$ is irreducible, according to the results of [KK79]. Therefore it is $\tilde{\mathfrak{n}}$-cofree, hence the result.

For an arbitrary $\kappa \neq \kappa_{\mathrm{c}}$ and $\lambda$ we use the same spectral sequence as in the proof of Theorem 6.2 induced by the PBW filtration on $\mathbb{M}_{\lambda, \kappa}$. Then the first term of this spectral sequence is the same as for $\kappa=\kappa_{\mathrm{c}}$, i.e. $H^{\bullet}\left(\widetilde{\mathfrak{b}}, \mathfrak{h}, \mathbb{M}^{\mathrm{cl}}\right)$. Next, we find that the first differential of this spectral sequence differs from the first differential of the corresponding spectral sequence at $\kappa=\kappa_{\mathrm{c}}$ by $h \operatorname{gr} \widetilde{\varphi}_{\kappa_{0}}^{i}$, 
where $\kappa=\kappa_{\mathrm{c}}+h \kappa_{0}$. But we know from the proof of Theorem 6.2 that the first differential of the spectral sequence at $\kappa=\kappa_{\mathrm{c}}$ is equal to 0 , and we know that $\widetilde{\varphi}_{\kappa_{0}}^{i}$ is just the de Rham differential on $H^{\bullet}\left(\widetilde{\mathfrak{b}}, \mathfrak{h}, \mathbb{M}^{\mathrm{cl}}\right) \simeq \Omega^{\bullet}\left(C_{\mathfrak{g}^{*}, \omega}^{\mathrm{RS}}\right)$. Hence we obtain the desired assertion.

\section{ACKNOWLEDGEMENTS}

We wish to thank B. Feigin and D. Gaitsgory for useful discussions. We are also grateful to D. Gaitsgory for his careful reading of the manuscript and his comments and suggestions.

The research of EF was supported by grants from DARPA and NSF, and the research of CT was supported by grants from NSF and EPSRC.

\section{REFERENCES}

BD05 A. Beilinson and V. Drinfeld, Quantization of Hitchin's integrable system and Hecke eigensheaves, Preprint (2005), available at www.math.uchicago.edu/ benzvi.

DS85 V. Drinfeld and V. Sokolov, Lie algebras and KdV type equations, J. Sov. Math. 30 (1985), 1975-2036.

Fei91 B. Feigin, Differential operators on the moduli space of G-bundles over curves and Lie algebra cohomology, in Special functions, eds M. Kashiwara and T. Miwa (Springer, Berlin, 1991), 90-103.

FF92 B. Feigin and E. Frenkel, Affine Kac-Moody algebras at the critical level and Gelfand-Dikii algebras, in Infinite analysis, eds A. Tsuchiya, T. Eguchi and M. Jimbo, Advanced Series in Mathematical Physics, vol. 16 (World Scientific, Singapore, 1992), 197-215.

FGT04 S. Fishel, I. Grojnowski and C. Teleman, The strong Macdonald conjecture and Hodge theory on the loop Grassmannian, Preprint (2004), math.AG/0411355.

Fre04 E. Frenkel, Wakimoto modules, opers and the center at the critical level, Adv. Math. 195 (2004), 297-404.

FB04 E. Frenkel and D. Ben-Zvi, Vertex algebras and algebraic curves, second edition, Mathematical Surveys and Monographs, vol. 88 (American Mathematical Society, Providence, RI, 2004).

Fuc88 D. B. Fuchs, Cohomology of infinite-dimensional Lie algebras (Plenum Press, London, 1988).

Hes76 W. H. Hesselink, Cohomology and the resolution of the nilpotent variety, Math. Ann. 223 (1976), 249-252.

KK79 V. Kac and D. Kazhdan, Structure of representations with highest weight of infinite-dimensional Lie algebras, Adv. Math. 34 (1979), 97-108.

Lan84 S. Lang, Algebra (Addison-Wesley, Reading, MA, 1984).

Str78 J. R. Strooker, Introduction to categories, homological algebra and sheaf cohomology (Cambridge University Press, Cambridge, 1978).

Edward Frenkel frenkel@math.berkeley.edu

Department of Mathematics, University of California, Berkeley, CA 94720, USA

Constantin Teleman teleman@dpmms.cam.ac.uk

DPMMS, Centre for Mathematical Sciences, Wilberforce Road, Cambridge CB3 0WB, UK 